\title{
The impact of contact angle on flow resistance reduction in hydrophobic micro pin fins
}

\author{
Ning Guan, Guilin Jiang, Zhigang Liu, Chengwu Zhang, Ning Ding
}

Energy Research Institute of Shandong Academy of Sciences, Jinan, 250014, Shandong, China

Corresponding author: Zhigang Liu, Tel:+86 0531 68606140; fax: +86 0531 82961954, E-mail address:

zgliu9322@163.com 


\title{
The impact of contact angle on flow resistance reduction in hydrophobic micro pin fins
}

\author{
Ning Guan, Guilin Jiang, Zhigang Liu*, Chengwu Zhang, Ning Ding \\ Energy Research Institute of Shandong Academy of Sciences, Jinan, 250014, Shandong, China
}

\begin{abstract}
The pressure drops and the friction factors are measured when ultra pure water flows through the elliptical, diamond and circular hydrophobic micro pin fins with different contact angles of $\theta=99.5^{\circ}, 119.5^{\circ}$ and $151.5^{\circ}$, and the coefficients of pressure drop reduction $d p_{-}$coe and friction factor reduction $d f_{-}$coe are calculated respectively. It is found that the value of $d p_{-} c o e$ becomes large with the increase of $\theta$ in elliptical and diamond micro pin fins, but for circular micro pin fins it increases at first and then decreases. With the increase of $\theta$, both values of $d p_{-}$coe and $d f_{-}$coe are reduced with the increase of the flow rate in elliptical micro pin fins, but the values are reduced at first and then become almost constants in diamond and circular micro pin fins. Besides, the minimal value of $d f_{\_}$coe is over $50 \%$ in diamond and circular micro pin fins with contact angle of $\theta=151.5^{\circ}$. When $\theta \geq 119.5^{\circ}$, the values of $d p \_c o e$ and $d f_{-}$coe in diamond and circular micro pin fins are both larger than those in elliptical test sections, but for $\theta=99.5^{\circ}$ the former are slightly smaller than the latter at $\operatorname{Re}<600$.
\end{abstract}

Keywords: hydrophobic micro pin fins, flow resistance reduction, contact angles, Reynolds number

\begin{tabular}{|c|c|c|c|c|c|}
\hline \multicolumn{6}{|c|}{ Nomenclature } \\
\hline$d$ & hydraulic diameter & $\mathrm{m}$ & $N$ & number of the rows & \\
\hline$d p \_c o e$ & $\begin{array}{l}\text { changing coefficient of } \\
\text { nraccura dman }\end{array}$ & & $N L$ & maximal number of the lines & \\
\hline$d f_{-} c o e$ & $\begin{array}{l}\text { changing coefficient of } \\
\text { friction factor }\end{array}$ & & $p$ & pressure & $\mathrm{Pa}$ \\
\hline$f$ & friction factor & & $\Delta p$ & pressure drop & $\mathrm{Pa}$ \\
\hline$G$ & flow rate of volume & $\mathrm{ml} / \mathrm{min}$ & $\operatorname{Re}$ & Reynolds number & \\
\hline$h$ & hgiht of the channel & $\mathrm{m}$ & $S$ & Distance between the pin fins & $\mathrm{m}$ \\
\hline$L$ & length of the channel & $\mathrm{m}$ & $u$ & velocity of fluid & $\mathrm{m} / \mathrm{s}$ \\
\hline$m$ & flow rate of mass & $\mathrm{kg} / \mathrm{s}$ & $W$ & $\begin{array}{l}\text { width of the channel/distance } \\
\text { between the nano projections }\end{array}$ & $\mathrm{m}$ \\
\hline \multicolumn{6}{|c|}{ Greek letters } \\
\hline$\phi$ & $\begin{array}{l}\text { angle between the } \\
\text { projections and the two } \\
\text { phase contact surface }\end{array}$ & & $\rho$ & water density & $\mathrm{kg} / \mathrm{m}^{3}$ \\
\hline$\gamma$ & Interfacial tension & $\mathrm{N} / \mathrm{m}$ & $\mu$ & water viscosity & $\mathrm{kg} /(\mathrm{m} \cdot \mathrm{s})$ \\
\hline$\theta$ & contact angle of water & & & & \\
\hline \multicolumn{6}{|c|}{ Subscript } \\
\hline air & air & & $\max$ & maximal value & \\
\hline$D$ & Inclined direction & & no-hy & $\begin{array}{l}\text { surfaces without hydrophobic } \\
\text { layers }\end{array}$ & \\
\hline
\end{tabular}

* Corresponding author, Tel:+86 0531 68606140; fax: +86 0531 82961954, E-mail address: zgliu9322@163.com 


\begin{tabular}{|llll|}
\hline$f$ & fin & $s l \quad \begin{array}{l}\text { Interface between the solid and } \\
\text { the liquid }\end{array}$ \\
$L$ & $\begin{array}{l}\text { Interface between the solid and } \\
\text { the vapor }\end{array}$ \\
$h v$ & $\begin{array}{l}\text { Interface between the } \\
\text { liquid and the vapor } \\
\text { surface with hydrophobic } \\
\text { layers }\end{array}$ & $T$ & Transverse direction \\
\hline
\end{tabular}

\section{O Introductions}

In recent decades, the Complementary Metal Oxide Semiconductor industries and other microelectronics industry have been developed rapidly. According to Moore's law, the component density and performance of integrated circuits will be doubled every 18 months [1], which results in an exponential increase of the heat flux in the smaller and smaller space. In order to maintain the operating temperature of the microelectronics elements, the investigation on micro cooling elements and heat sinks becomes one of the most important topics in flow and heat transfer. Many heat sinks with micro scale structures are explored by many researchers in these years. The micro pin fin heat sink is one of the most potential cooling structures due to the high cooling capacity [2-3], thus many researchers carried out investigations on the flow and heat transfer characteristics in micro pin fins. These investigations verify the cooling capacity of micro pin fins, but the flow resistance through the micro pin fins is so high that it hinders the further development of the micro pin fin heat sinks in the cooling system of microelectronic elements.

Many literatures explored the reduction of flow resistance in micro pin fins by optimizing the geometry or changing the working fluid. According to the hydrodynamic theories, the reduction of the surface energy on the flowing surface can be employed to reduce the flow resistance in micro/nano channels due to the appearance of a thin layer of liquid with low density near the wall [4-7], so it may be an effective way to reduce the high pressure drop and flow resistance in micro pin fins. Therefore, many researchers have investigated the influence of hydrophobic surface on the flow and heat transfer characteristics in micro channels.

Three topics have been mentioned in the existing literatures about the flow and heat transfer in hydrophobic micro channels: the single flow characteristics and the resistance reduction in channel with hydrophobic surfaces, the effect of hydrophobic characteristics on the mass transfer in porous media, and the condensation heat transfer in hydrophobic channels.

As mentioned above, the researches of single phase flow confirm the flow resistance reduction in micro channels with hydrophobic surfaces. Ou J. [8-9] carried out a series of experiments to study the flow kinematics of water past drag-reducing super-hydrophobic surfaces. They fabricated ultra-hydrophobic surfaces from silicon wafers using photolithography, and experimentally measured the velocity profile and the pressure drop as a function of the flow rate for a series of rectangular cross-section micro-channel geometries and ultra-hydrophobic surface designs. The results demonstrated that the primary mechanism responsible for the drag reduction was the flow slip along the shear-free air-water interface supported between the hydrophobic micrometer-sized ridges in the flow on ultra-hydrophobic surfaces. A maximum slip velocity of more than $60 \%$ of the average velocity in the micro-channel was found at the center of the shear-free air-water interface whereas the no-slip boundary condition was found to hold along the surface of the hydrophobic ridges. Besides, their investigations showed that pressure drop reductions up to $40 \%$ and apparent slip lengths larger than $20 \mu \mathrm{m}$ were obtained using ultra-hydrophobic surfaces. In order to clarify the slip flow in hydrophobic in micro channels, Tretheway D. C. [10] carried out experiments with micro-PIV technology to measure the velocity profiles of water flowing through $30 \times 300 \mu \mathrm{m}$ 
channels. The velocity profiles were measured in the zones within $450 \mathrm{~nm}$ from the micro-channel surface, and the results showed that an apparent velocity slip was measured just above the solid surface. This velocity was approximately $10 \%$ of the free-stream velocity and yielded a slip length of approximately $1 \mu \mathrm{m}$. For this slip length, slip flow was negligible for length scales greater than $1 \mathrm{~mm}$, but it must be considered at the micro and nano scales. Markus Hilpert M. [11] carried out analytical investigations on the effects of dynamic contact angle (contact angle of water on the surface of a capillary tube when the water flows through the tube) on liquid infiltration into inclined capillary tubes, and obtained an analytical solution for travel time (the average time between successive collisions of the gas molecules) as a function of interface position. Mohammad N. N. [12] presented a theoretical prediction of friction drag reduction in turbulent channel flow with super-hydrophobic surfaces. The predicted drag reduction was approximately $30 \%$, which concurred with results obtained from Direct Numerical Simulation (DNS). An important implication of the present finding was that the near-wall turbulence structures were modified with stream wise slip velocity. In addition, a noticeable effect on the turbulence structure occurred when the slip length was greater than a certain value. Yu D. [13] measured the pressure drops of slugs with triple-lines for various diameters, fluids, and a range of velocities $(0.01-0.4 \mathrm{~m} / \mathrm{s})$. Dynamic contact angles were calculated from an equation describing the pressure drop of a triple-line, and it was found that the previous correlations had underestimated the dynamic contact angles in comparison with the experimental values, and then a new dynamic contact angle correlation for a regime was proposed. Lyua S. [14] employed processes with the advantages of simplicity and cost effectiveness to obtain durable super-hydrophilic and super-hydrophobic surfaces, and the results of measurements on a super-hydrophobic surface were compared to those on smooth and super-hydrophilic surfaces. The experimental results illustrated that the flow resistance in micro channels could be reduced apparently at $R e<200000$, especially at low $R e$. Wang B. [15] designed the transverse grates to be dense and deep to sustain air pockets in the gaps of hydrophobic grooves for a long time. Direct optical measurements were conducted to observe the entrapped gas when water flowed over the surface in the perpendicular direction of grating pattern. Visualization of gas indicated that the gas could be held in the designed structures within water flowing time. When grooves were optimized, a drag reducing efficiency of more than $13 \%$ was achieved, which did not vary during the test lasting $1 \mathrm{~h}$. The drag reduction mechanism of this specially designed surface was attributed to an "effective" slip which was generated by the steady gas in the microgrooves underwater. Li B. W. [16] simulated the characteristics of flow in a micro-channel with patterned super-hydrophobic surfaces by using an incompressible lattice Bhatnagar-Gross-Krook (LBGK) model. They found that the depth-to-width ratio of the cavities between adjacent micro-ridges was an important effect parameter for the flow in the micro-channel. Mastrokalos M. E. [17] performed a computational investigation of flow past a circular cylinder with slip conditions at low $R e$, and the results showed that a proper use of partial hydrophobic surface could lead to a significant reduction in passive control effort for both full and partial suppression of the Karman vortex street, and the flow stabilization was attained when a global intensity of absolute instability was sufficiently reduced.

Except for the single phase flow in channels, the hydrophobic surface also affects the flow performance in porous media. For an instance, the gas diffusion layer (GDL) in fuel cell and the metal meshes with hydrophobic properties was also researched. Cai Y. H. [18] simulated the mobility of water droplets and water films inside a straight micro-channel of a proton exchange membrane fuel cell to study the effects of the hydrophilic/hydrophobic properties on water behavior. The results showed that water moved faster on a hydrophobic surface, and water and gas distribution under this condition was advantageous for water discharge and gas diffusion. Yin Y. [19] numerically investigated the two phase cross flow in micro structures of gas diffusion layer with variable contact angle, it was found that water transport characteristics in different cross sections normal to the through-plane direction with different contact angles were similar to the corresponding fixed contact angle cases in these cross sections, and the overall process of water intrusion with variable contact angle was similar to its corresponding average fixed contact angle case for a GDL with variable contact angle. 
Song M. [20] numerically investigated the droplet dynamics in the serpentine flow channel of a hydrogen fuel cell to obtain ideas for designing a serpentine channel with the aim of effectively preventing flooding. The numerical results showed that the hybrid case, which consisted of hydrophilic channel walls at the straight part and hydrophobic walls at the turning part of the serpentine flow channels, enhanced water removal compared with two other cases in which the channel wall was homogeneously hydrophilic or hydrophobic. Avcioglu G. S. [21] investigated physical and electrochemical characterization of catalyst layers with hydrophobic nanoparticles and carried out performance tests. Sang Y. C. [22] investigated the properties of copper and stainless steel meshes, coated with super hydrophobic electrolessly deposited silver, and the results showed that copper mesh and stainless steel meshes with the same pore size had almost the same contact angle.

The condensation heat transfer enhancement with hydrophobic surfaces has also been researched by many literatures. Lenzinger M. [23] introduced an approximation procedure and provided existence results for two-phase flow equations in porous media that had hydrophobic and hydrophilic components. The capillary pressure function was degenerate for extreme saturations, and an approximate system introducing standard boundary conditions was presented in this research, which can be used in numerical schemes and allowed the derivation of maximum principles. Choi C. [24] conducted experiments of water flow boiling in hydrophilic and hydrophobic rectangular micro-channels to investigate the wet ability effect on flow boiling in rectangular micro-channels. The boiling heat transfer coefficient in the hydrophobic rectangular micro-channel was higher than that in the hydrophilic rectangular micro-channel, which was highly related with nucleation site density and liquid film motion. And the pressure drop in the hydrophobic rectangular micro-channel was higher than that in the hydrophilic rectangular micro-channel. Liu T. Y. [25] fabricated three different micro-channels with identical sizes at $105 \times 1000 \times 30000 \mu \mathrm{m}$ but at different wet ability of $36^{\circ}$ and $103^{\circ}$. In addition, a vapor-liquid-solid growth process was adopted to grow nanowire arrays on the wafer so that the surface became super-hydrophilic with a contact angle close to 0. Periodic oscillation in temperatures was not found in both the hydrophobic and the super-hydrophilic surface. During the experiments, the heat flux imposed on the wall varied from 230 to $354.9 \mathrm{~kW} / \mathrm{m}^{2}$ and the flow of mass flux into the channel from 50 to $583 \mathrm{~kg} / \mathrm{m}^{2} \mathrm{~s}$. Detailed flow regimes in terms of heat flux versus mass flux were also obtained. Cho S. C. [26] investigated two-phase flows in a micro channel with hydrophilic and hydrophobic surfaces both experimentally and numerically. It was observed that the surface wetting property and roughness affected the presence site of liquid water flow: liquid water was preferentially present in the two hydrophilic corners when one channel wall was hydrophobic. As a result, two-phase pressure, patterns, and stability differed from the purely hydrophilic channel. Rough surface was found to affect two-phase flow. Derby M. M. [27] investigated the enhancement of flow condensation of steam on hydrophobic and hydrophilic surfaces, and it was found that enhancement factors of 3.2-13.4 times that of the hydrophilic channel.

It can be known from the above literatures that the wet ability of the surface is proved to be an important factor to impact the flow characteristics in micro channels, but the flow resistance reduction in hydrophobic micro pin fin sinks has not been clear yet. Especially for the hydrophobic micro pin fins with different cross section shapes, the optimization of contact angles for the resistance reduction should be explored.

In present research, the experimental investigations are carried out to study the flow resistance reduction in hydrophobic micro pin fin heat sinks with different contact angles of $99.5^{\circ}, 119.5^{\circ}$ and $151.5^{\circ}$, respectively, and the pressure drops, the friction factors are measured in micro pin fins with cross section shapes of ellipse, diamond and circle. Based on the experimental results, the reduction coefficients of pressure drops and friction factors in micro pin fins with different contact angles are obtained to analyze the mechanisms of resistance reduction in micro pin fins with hydrophobic surfaces and different cross section shapes.

\section{Preparation of the test sections and the experimental measurements}

\subsection{Preparation of the hydrophobic micro pin fins with different contact angles}


Hydrophobic solution is prepared by adding $2 \%$ perfluorinated octyl fluorine silane and micro-nano particles to modified silicone dilute solution, and this solution is sprayed on the surfaces of staggered arranged copper micro pin fins to obtain the hydrophobic test sections, then the test sections are placed into a vacuum container with temperature of $80^{\circ}$ to solidify the hydrophobic layer on the flowing surfaces in the micro pin fins. Different contact angle of the hydrophobic surface can be obtained by adjusting the content of micro-nano particles in hydrophobic solution.

In the spraying process, a high pressure airbrush is used to atomize the hydrophobic solution, and the tilted airbrush sprays the hydrophobic solutions on the test sections that rotate uniformly to obtain a hydrophobic surface. The contact angles of six different locations on the surface of the test section with a solidified hydrophobic layer are measured, and the discrepancies of contact angles among these measurements are less than $\pm 1^{\circ}$.

Employing the preparation method above to the elliptical, diamond and circular micro pin fins, the hydrophobic test sections are obtained with contact angles of $\theta=99.5^{\circ}, 119.5^{\circ}$ and $151.5^{\circ}$. The contact angles of hydrophobic surfaces are measured by the contact angle measuring instrument JC-2000 (Chenguang Ltd, Shanghai), and the photos of measurements are shown in Fig.1.

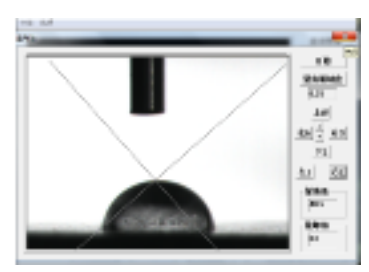

(a) $83^{\circ}$ (blank)

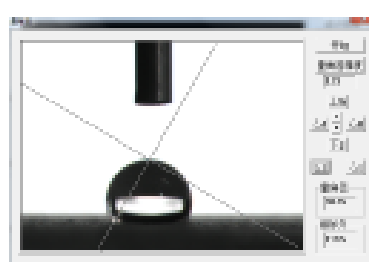

(c) $119.5^{\circ}$

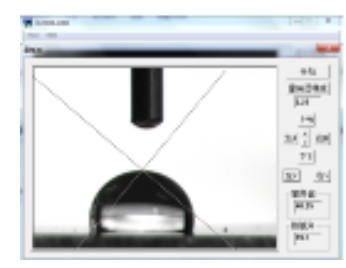

(b) $99.5^{\circ}$

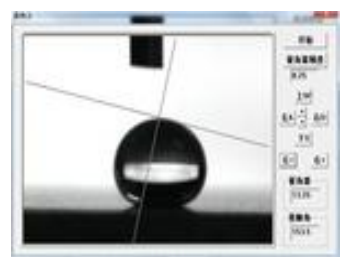

(d) $151.5^{\circ}$

Fig.1 Contact angle measurements on the ordinary copper surfaces and the hydrophobic surfaces

The photos and the schematics of the micro pin fins investigated in present research are shown in Fig.2, and the dimensions of these test sections are listed in Tab. 1. It is known from Tab.1 that the dimensions of the micro pin fins are so small that the thickness of the hydrophobic layer on the surface may change the characteristic dimensions of the pin fins significantly. In order to clarify the change of the dimensions of the micro pin fins after the hydrophobic layer solidification, the thickness of the hydrophobic layers on the micro pin fins with different contact angles are measured by the scanning electronic microscope (SEM) and the photos are shown in Fig.3. The SEM measurements are repeated many times to choose the test sections with diameter discrepancies among the pin fins smaller than $\pm 0.001 \mathrm{~mm}$. The copper test sections shown in Fig. 2 with staggered micro pin fins are manufactured by numerical control machine P25C/CA (KUHLMANN Co Ltd, Germany), which can precisely control the dimension of the test section with an accuracy of $\pm 1 \mu \mathrm{m}$.

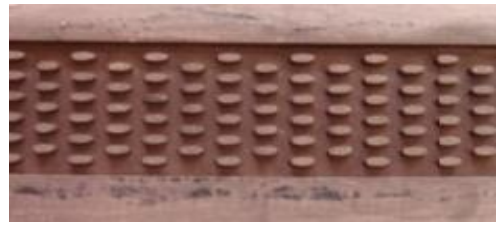

(a)

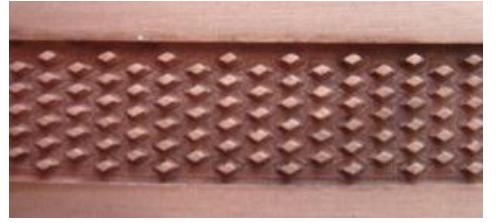

(b)

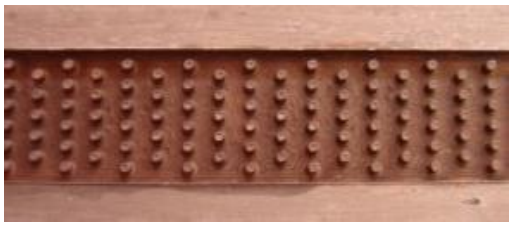

(c) 


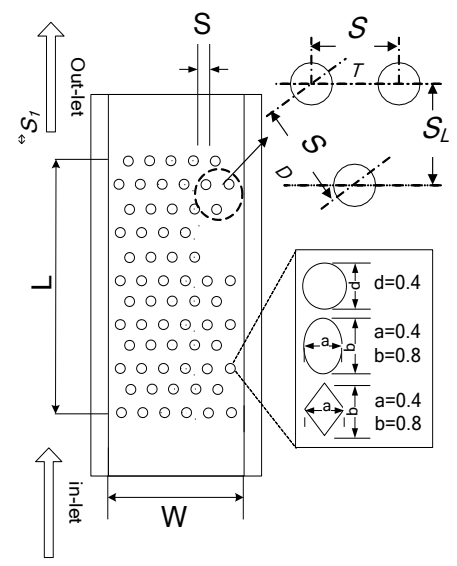

(d)

Fig.2 Photos of test sections

Tab.1

\begin{tabular}{|c|c|c|c|c|c|c|}
\hline Cross section shapes & $\begin{array}{c}h \\
(\mathrm{~mm})\end{array}$ & $\begin{array}{c}d \\
(\mathrm{~mm})\end{array}$ & & $\begin{array}{c}S_{T} \\
(\mathrm{~mm})\end{array}$ & $\begin{array}{c}S_{L} \\
(\mathrm{~mm})\end{array}$ & $\begin{array}{c}S_{D} \\
(\mathrm{~mm})\end{array}$ \\
\hline Ellipse & 0.5 & $\begin{array}{l}\text { semimajor : } \\
\text { semiminor: } 0.2\end{array}$ & 0.4 & 0.8 & 1.2 & 1.265 \\
\hline Diamond & 0.5 & $\begin{array}{l}\text { Long axle: } \\
\text { Short axle: } 0.4\end{array}$ & 0.8 & 0.8 & 1.2 & 1.265 \\
\hline Circle & 0.5 & 0.4 & & 0.8 & 1.2 & 1.265 \\
\hline
\end{tabular}

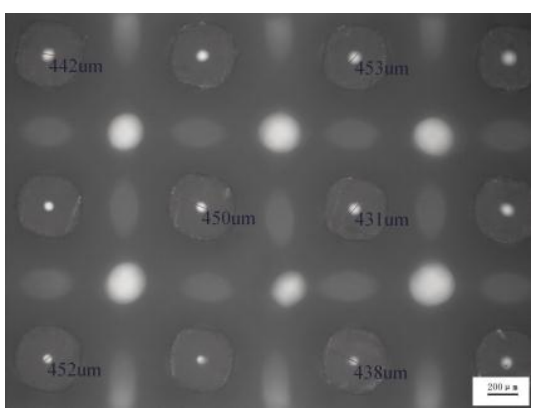

(a) $d=0.441 \mathrm{~mm}, \theta=99.5^{\circ}$

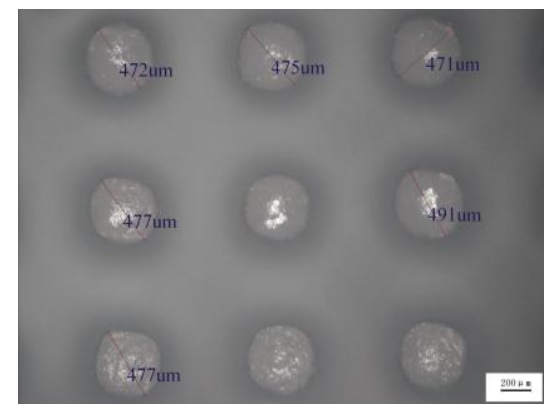

(b) $d=0.489 \mathrm{~mm}, \theta=119.5^{\circ}$

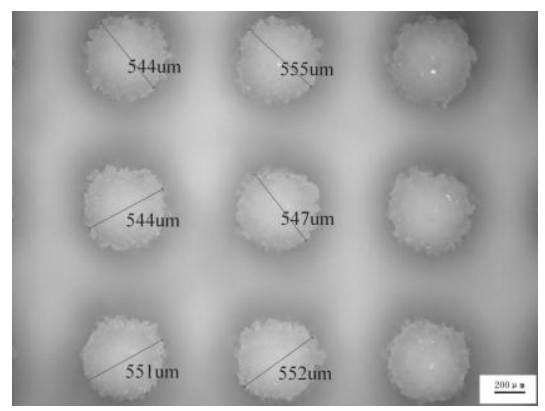

(c) $d=0.551 \mathrm{~mm}, \theta=151.5^{\circ}$

Fig.3 Diameter measurement of micro pin fins with different contact angles by SEM

\subsection{The experimental setup and procedure for flow resistance measurements}

Experimental investigations are carried out to explore the flow resistance reduction in hydrophobic micro pin fins with different contact angles in present research, and the experimental loop is schematically shown in Fig.4.

As shown in Fig.4, the experimental apparatus is consisted of a pressure supply system, a liquid pool, a test section, and a data acquisition system, etc. The stable and exact pressure nitrogen is supplied by the pressure supply system composed by a nitrogen bottle, a three-layer filter, a precise pressure regulating valve and a quick-opening valve. The nitrogen bottle with maximum pressure of 12MPa maintains a given pressure (less than 1.6MPa) precisely with control of a precise pressure regulating valve. In order to prevent impurities in the nitrogen flowing into the test section, a three-filter with the aperture of filtration film of $50 \mu \mathrm{m}, 25 \mu \mathrm{m}$ and $5 \mu \mathrm{m}$ in turn is installed between the nitrogen bottle and the precise pressure regulating valve. A gas storage reservoir is used in the experimental system to 
avoid gas fluctuation. Working fluid of distilled water flows out of the liquid pool and flows through the test section. A polyurethane tube with 2MPa resisting pressure connects the liquid pool to a joint just upstream of the micro tube, and the cross-sectional area of the tube is over 30 times of the largest hydraulic diameter of the micro pin fins, which ensures that the fluid velocity in the polyurethane tube was negligible small. The fluid flow rate for a given pressure difference and a test section is determined by a flow meter. The measurement is initiated after the prescribed experiment pressure is reached by opening the reducing valve in the nitrogen bottle and adjusting the precise regulating valve. When the flow rate read from the flow meter reaches a stable pre-set value, the experimental data should be recorded.

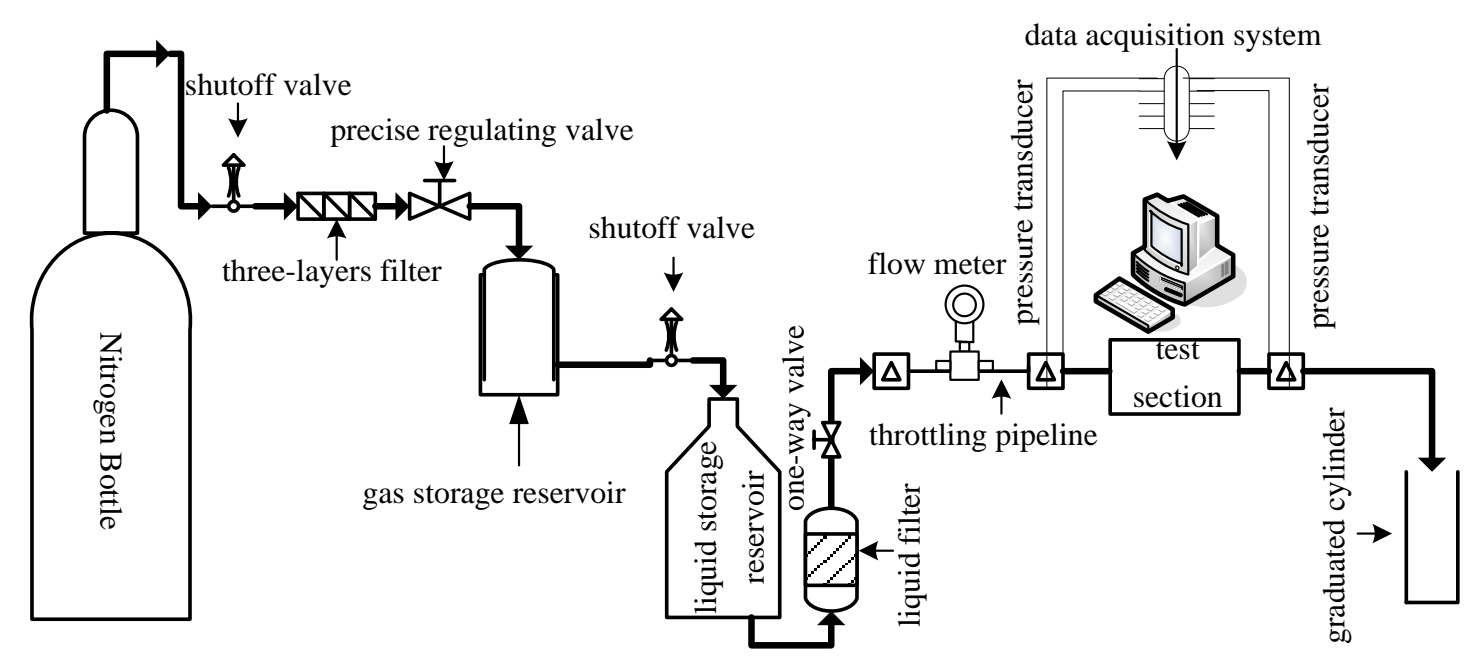

Fig. 4 Experimental loop of micro pin fin flow and heat transfer

In the present experiment, the main uncertainties come from the measurements of the temperature, the pressure drop, the flow rate and the geometrical dimensions of micro pin fins. The uncertainty of the pressure is less than $\pm 1 \mathrm{kPa}$ for the pressure transducers of CYB-10S type (CASC, Beijing); the accuracy of flow meter using to measure the flow rate of the working fluid is $\pm 0.1 \mathrm{mg} / \mathrm{s}$. The uncertainties of geometrical dimensions of test sections are $\pm 20 \mathrm{~nm}$ depending on the accuracy of the SEM.

The experimental uncertainties mentioned above are calculated by the error analysis method in reference literature [29] and listed in Table 2.

Tab.2 Experimental uncertainties

\begin{tabular}{lll}
\hline$\square p$ & $R e$ & $f$ \\
\hline $\pm 0.2 \%$ & $\pm 2.9 \%$ & $\pm 5.7 \%$ \\
\hline
\end{tabular}

In present research, the largest ratio of chord length to the span length of the pin fin is 1.5 , so the span length should be considered in the calculation of the characteristic dimension of the micro pin fins. Besides, the results of Kosar [30] showed that the hydraulic diameter of micro pin fins could be used as characteristic dimension to calculate the friction factors, thus the present work uses the hydraulic diameters of micro pin fins as the characteristic dimensions.

The $R e$ can be expressed by the following equation:

$$
R e=\frac{\rho u_{\max } \cdot d}{\mu}
$$

Where, $u_{\max }$ is the maximal flow velocity in flow channel, and it is obtained by: 


$$
u_{\max }=\frac{m}{\rho h\left(W-N L^{*} d\right)}
$$

The friction factor for three test sections can be obtained by the following equation:

$$
f=\frac{2 \Delta p}{\rho u_{\max }^{2} N}
$$

For all of the test sections, the entrance lengths in channels are very short, while the pressure drops of liquid flow through the micro pin fins are much higher that those past the entrance without pin fins. Therefore, the entrance effect on pressure measurements is ignored in present investigation.

In order to analyze the influence of super-hydrophobic surfaces on the pressure drops and the friction factors in micro pin fins with different contact angles and cross section shapes, two parameters are defined in this manuscript to evaluate the resistance reduction. One is the coefficient of pressure drop reduction which is defined as follows:

$$
d p_{-} \operatorname{coe}=\frac{\Delta p_{n o-h y}-\Delta p_{h y}}{\Delta p_{n o-h y}}
$$

Another is the coefficient of friction factor reduction defined as Eq.(5):

$$
d f_{-} c o e=\frac{f_{n o-h y}-f_{h y}}{f_{n o-h y}}
$$

\section{Results and discussions}

\subsection{Effects of the contact angle on the flow resistance characteristics}

Based on the experimental measurements, the flow resistance characteristics in micro pin fins with different contact angles are obtained. In order to analyze the influence of the contact angle on the pressure drops in micro pin fins, the profiles of pressure drops vs. $G_{\mathrm{v}}$ are shown in Fig.5 in elliptical, diamond and circular micro pin fins with volume flow rate ranged from $20-240 \mathrm{ml} / \mathrm{min}$.

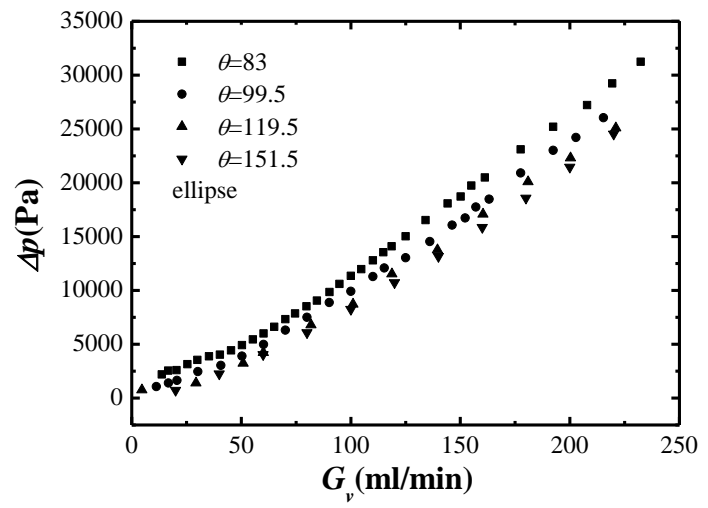

(a) ellipse

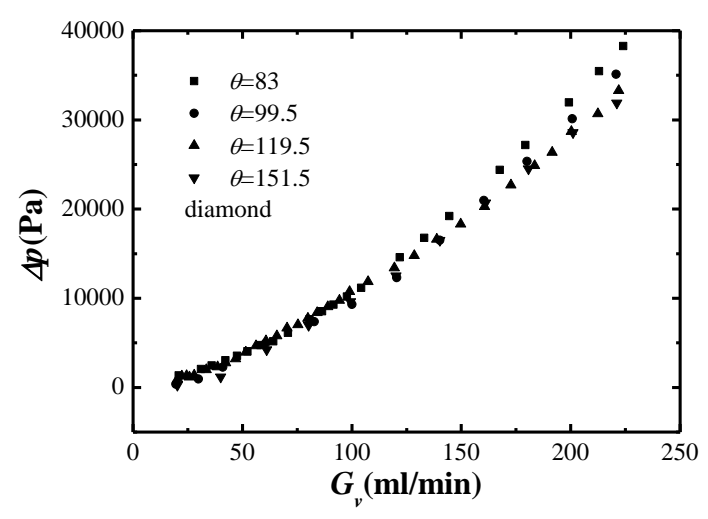

(b) diamond 


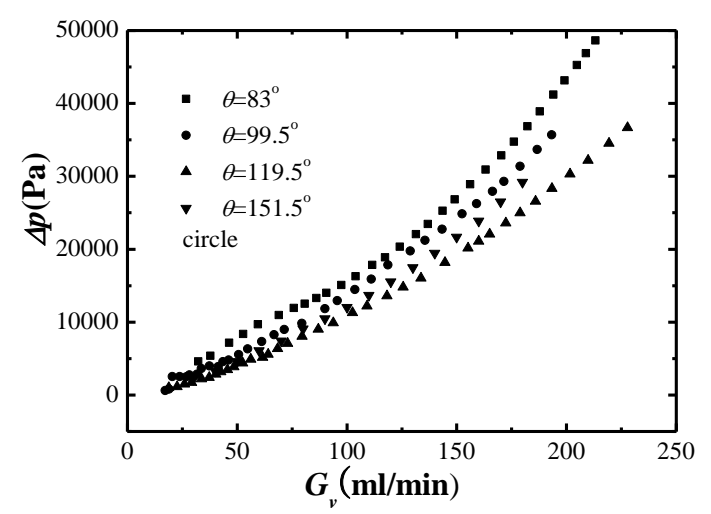

(c) circle

Fig.5 Profiles of pressure drops vs. volume flow rate in micro pin fins with different contact angles

It can be known from Fig.5 (a) that the pressure drop of liquid water flowing through the elliptical micro pin fins is reduced with the increase of the contact angle at the same volume flow rate, which is attributed to the flow resistance reduction of hydrophobic surfaces with large contact angle. In present research, a hydrophobic layer with nano/micro particles is solidified on the surface of the micro pin fins to obtain a hydrophobic surface with a particular microstructure, and the SEM photos of the hydrophobic surfaces are shown in Fig.6(b). It can be seen that the hydrophobic surface is composed by huge numbers of the nano scale projections that shaped the micro pin fin surfaces similar to the hydrophobic model of Ou J. [8-9], as shown in Fig.6(a). According to Ou J.'s model, the flow resistance on a hydrophobic surface is reduced due to flow slip on the air-water interface, as shown in Fig.6(a)-(b). Besides, the change of the forces on the hydrophobic surface is another reason to reduce the flow resistances. Figures 6(c)-(d) illustrate the force diagrams on the pin fin with hydrophobic and hydrophilic surface, and it is known from the figures that the flow resistance is reduced by the interfacial tension distribution on the surface with $\theta>90^{\circ}$ when the liquid water flows across the pin fin due to the differential pressure and the frictional shear stress, as shown in Fig.6(c). Meanwhile, the flow resistance across the pin fin with $\theta<90^{\circ}$ increases since the frictional shear stress increases due to the component of interfacial tension $\gamma_{\mathrm{v}}$ in the tangential direction, as shown in Fig.6(d). Therefore, the pressure drop in micro pin fins with large contact angle is smaller than that in micro pin fins with $\theta=83^{\circ}$.

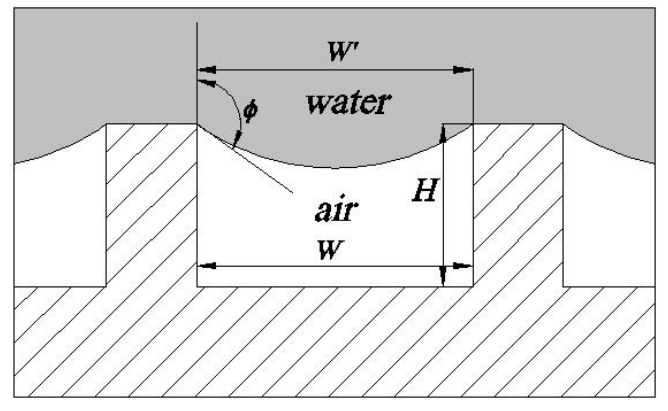

(a) schematic of Ou's model

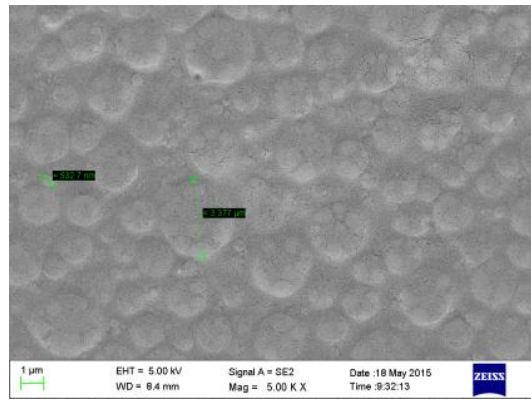

(b) photo of SEM 


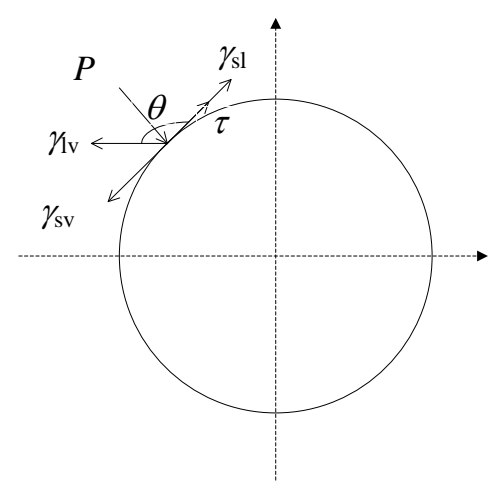

(c) force diagram on the hydrophobic surface

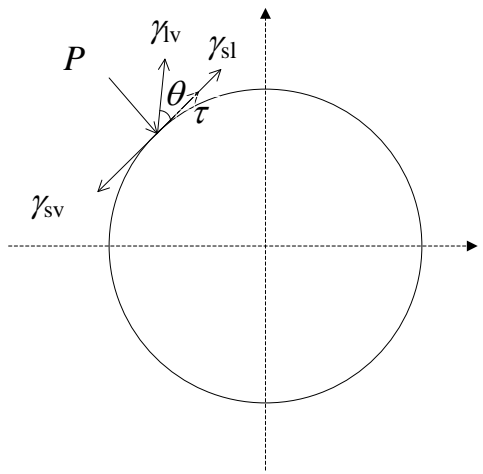

(d) force diagram on the hydrophilic surface

Fig.6 Schematic diagram of model for hydrophobic drag reduction and the force diagram on the pin fin surface

The surface tension on the interfaces of the microstructures prevents the water intruding into the gaps among the nano projections, and thus the air-water two-phase flow occurs in the microstructures of the hydrophobic surfaces in micro pin fins. The shear stress is reduced by the slip flow on the air-water interfaces between the liquid and the solid wall in the flow, so the flow resistance and the pressure drop are apparently reduced by increasing the contact angle. Although the characteristic dimensions of the micro pin fins become large with the increase of the contact angle due to the thickness of the solidified hydrophobic layer with nano particles, the effect of the characteristic dimension change on the pressure drop is less than the pressure drop reduction due to the increase of the contact angle in elliptical micro pin fins, as shown in Fig.5 (a).

For the diamond micro pin fins, as shown in Fig.5(b), the pressure drop is reduced apparently with the contact angle increasing from $\theta=83^{\circ}$ to $\theta=119.5^{\circ}$. Keep increasing the contact angle of diamond micro pin fins from $\theta=119.5^{\circ}$ to $\theta=151.5^{\circ}$, the pressure drop is also reduced at low flow rate in diamond micro pin fins, however the pressure drop with $\theta=151.5^{\circ}$ is close to those of $\theta=119.5^{\circ}$ when $G_{v}>150 \mathrm{ml} / \mathrm{min}$. For the circular micro pin fins, the pressure drop is reduced by increasing the contact angle from $\theta=83^{\circ}$ to $\theta=119.5^{\circ}$, as shown in Fig.5(c), and the discrepancy of the pressure drop between circular micro pin fins of $\theta=119.5^{\circ}$ and $\theta=151.5^{\circ}$ is very different from those of elliptical and diamond micro pin fins. As shown in Fig.5(c), the pressure drop with $\theta=151.5^{\circ}$ is nearly equal with that of $\theta=119.5^{\circ}$ in circular micro pin fins at low flow rate, but the former becomes larger than the latter when $G_{v}>80 \mathrm{ml} / \mathrm{min}$. This phenomenon is attributed to two reasons: the first is the different mechanism of pressure drop reduction due to the hydrophobic surfaces in the micro pin fins with different cross section shapes. And the second is the characteristic dimension changes of the micro pin fins with the increase of the contact angles. It is known that the pressure drop in the flow across the micro pin fins is composed by the frictional resistance and the pressure drag, and the frictional resistance is the dominant in the flow with low velocity or across a well streamlined fin pin, because the boundary layer separation occurs lately and the wake zone area is relatively small. However, the area of wake zone is enlarged and the pressure drag gradually increases with the increase of the flowing velocity. Especially for the micro pin fins with poor streamlined shape such as the circular micro pin fins, the pressure drag plays more important role in the total pressure drop than that in the elliptical and diamond micro pin fins at the same flowing velocity. As mentioned above, the hydrophobic layer solidified on the micro pin fins reduces the shear stress near the flowing surface, but the thickness of the hydrophobic layer results in a change of the characteristic dimension of the pin fins. In addition, the the hydrophobic layer becomes thick with the increase of the contact angle, and the characteristic dimension of the circular micro pin fins changes $7.5 \%$ when the contact angle increases from $\theta=83^{\circ}$ to $\theta=99.5^{\circ}$, but the characteristic dimension with contact angle of $\theta=151.5^{\circ}$ is $37.75 \%$ higher than that of $\theta=83^{\circ}$ and $12.68 \%$ higher than that of $\theta=119.5^{\circ}$. The increase of the characteristic dimension results in not only the increase of the frictional resistance, but also the increase of the pressure drag due to the change of the flow velocity, especially for the circular micro pin fins. Although the slip flow near the wall reduces the shear stress near the wall when the contact angle increases from $\theta=119.5^{\circ}$ to $\theta=151.5^{\circ}$ in circular micro pin fins, 
the negative effect of the characteristic dimension change on the pressure drop is more apparent. Therefore, the pressure drop becomes large with the contact angle increasing from $\theta=119.5^{\circ}$ to $\theta=151.5^{\circ}$ in circular micro pin fins. Meanwhile, the pressure drop in diamond micro pin fins hardly changes when contact angle increases from $\theta=119.5^{\circ}$ to $\theta=151.5^{\circ}$, but for the elliptical micro pin fins the pressure drop always becomes small with the increase of the contact angle due to the dominant frictional resistance in the flow across the well streamlined pin fins, as shown in Fig.5 (a)-(c).

Figure 7 illustrates the relationship between the coefficient of pressure drop reduction with the volume flow rate when the contact angle on the surfaces of elliptical, diamond and circular micro pin fins changes from $\theta=83^{\circ}$ to $\theta=99.5^{\circ}, 119.5^{\circ}$ and $151.5^{\circ}$, respectively.

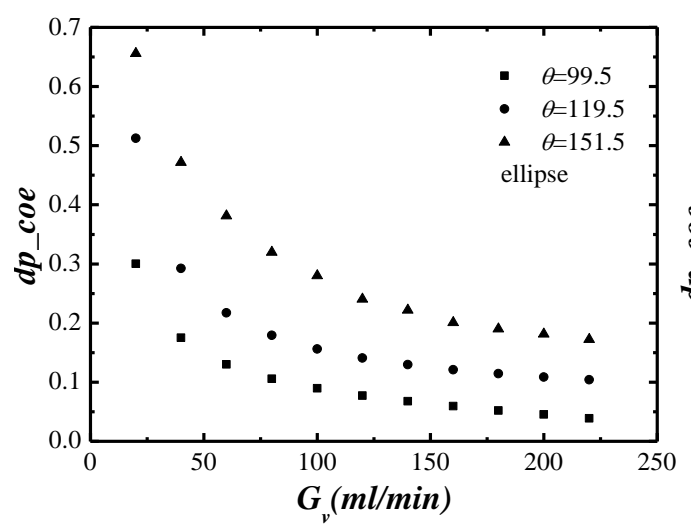

(a) elliptical micro pin fins

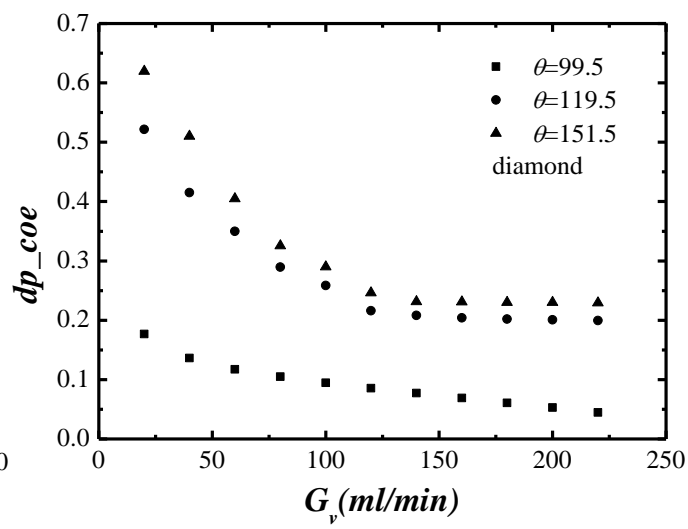

(b) diamond micro pin fins

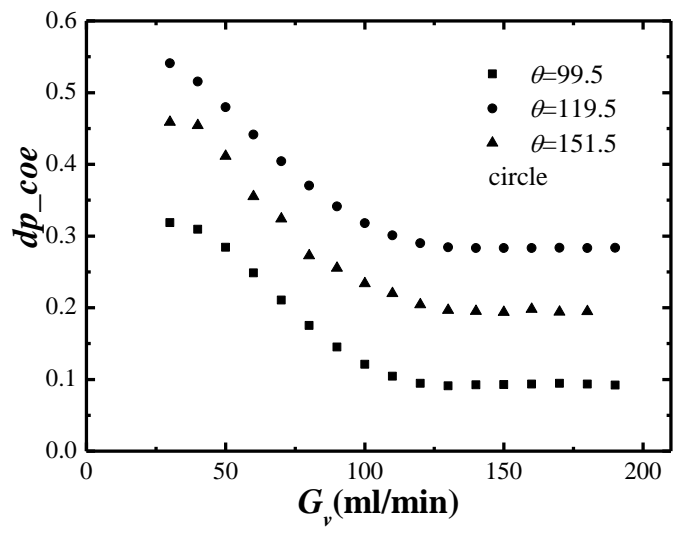

(c) circular micro pin fins

Fig.7 The relationships between the coefficient of pressure drop reduction with the volume flow rate in micro pin fins with contact angles

As discussed above, the shear stress near the wall is reduced by the flow slip on the hydrophobic surfaces with nano scale projections in micro pin fins with solidified hydrophobic layers. Consequently, the frictional resistance is reduced. For the elliptical micro pin fins with very well streamlined shapes, the coefficient of the pressure drop becomes large with the increase of the contact angle, as shown in Fig.7(a), the value of $d p \_c o e$ in elliptical micro pin fins with $\theta=151.5^{\circ}$ is apparently larger than that with smaller contact angle. Besides, the values of $d p \_c o e$ become small with the increase of flow rate in all of elliptical micro pin fins with three different contact angles. For an example, the value of $d p_{-} c o e$ is $41.51 \%$ reduced in elliptical micro pin fins with $\theta=151.5^{\circ}$ when the flow rate increases from $20 \mathrm{ml} / \mathrm{min}$ to $220 \mathrm{ml} / \mathrm{min}$. This phenomenon is attributed to the change of the inertial force and the surface tension in hydrophobic micro pin fins with the increase of flow velocity. For the micro pin fins with a certain contact angle, the surface tension is constant but the differential pressure on the air-water interface changes with the increase of the flowing velocity and the pressure of the working fluid. According to Ou J.'s model, the 
liquid water can not penetrate the gaps among the nano projections at low velocity and small differential pressure of the flow, thus the water-solid contact surfaces mainly equals with the total area of the nano projections. However, the pressure of the working fluid in the hydrophobic micro pin fins becomes large with the increase of the flow rate, as a result the differential pressure on the air-water interface becomes larger than the surface tension, and thus the working liquid gradually penetrates the gaps among the nano projections on the hydrophobic surfaces. As a result, the total area of the water-wall interface area is enlarged, and the pressure drop reduction is reduced in hydrophobic micro pin fins. For the diamond micro pin fins, the value of $d p_{-} c o e$ with $\theta=119.5^{\circ}$ is similar to that of $\theta=151.5^{\circ}$, but for the circular micro pin fins, the value of $d p_{-} c o e$ with $\theta=151.5^{\circ}$ is a little larger than that of $\theta=119.5^{\circ}$, as shown in Fig.7 (b)-(c), which is attributed to the change of the pressure drag and the characteristic dimensions with the increase of the contact angle in micro pin fins as discussed above.

Furthermore, it also can be found from the comparisons in Fig.7 that the value of $d p \_c o e$ always decreases with the increase of the flow rate in elliptical micro pin fins, but for the circular and the diamond micro pin fins it decreases first and then becomes a constant, especially in the micro pin fins with large contact angles. This may be attributed to that pressure drag is dominant in pressure drop of circular and diamond micro pin fins compared with the elliptical micro pin fins, and it is not influenced by the flow velocity and the pressure as much as the frictional resistance. Besides, the pressure near the wall in wake zones and near the separation location are almost equal with each other, so the impact of the increased inertial force on the frictional resistance reduction is reduced attributed to the small pressure and the velocity gradient in these regions in hydrophobic micro pin fins. The minimal value of $d p \_c o e$ turns out to be $22.92 \%$ and the corresponding contact angle is $\theta=151.5^{\circ}$ in diamond micro pin fins at high flow rate, but it is $28.35 \%$ when the contact angle is $\theta=119.5^{\circ}$ for the circular micro pin fins.

The changing relationships between the friction factors with $R e$ are shown in Fig.8 in micro pin fins with contact angles of $83^{\circ}, 99.5^{\circ}, 119.5^{\circ}$ and $151.5^{\circ}$.

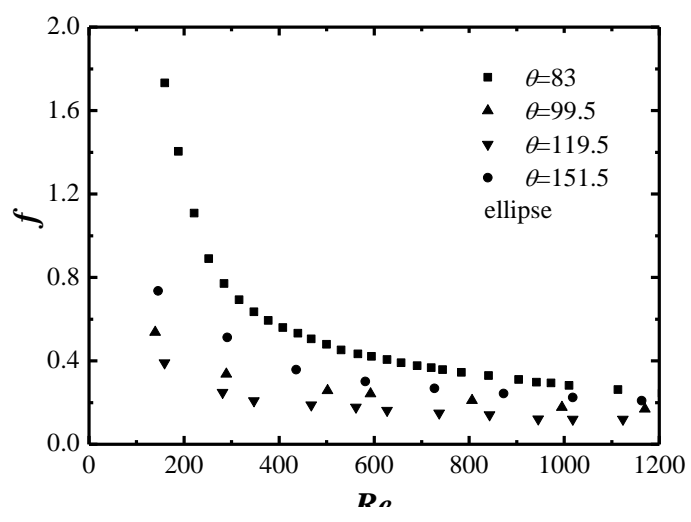

(a) elliptical micro pin fins

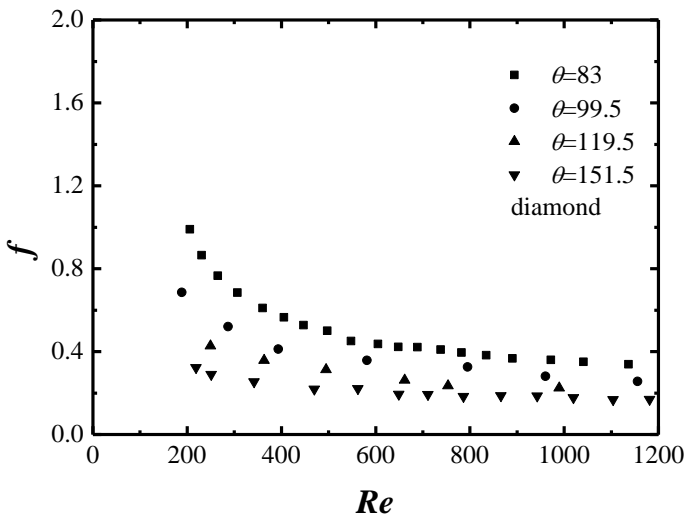

(b) diamond micro pin fins

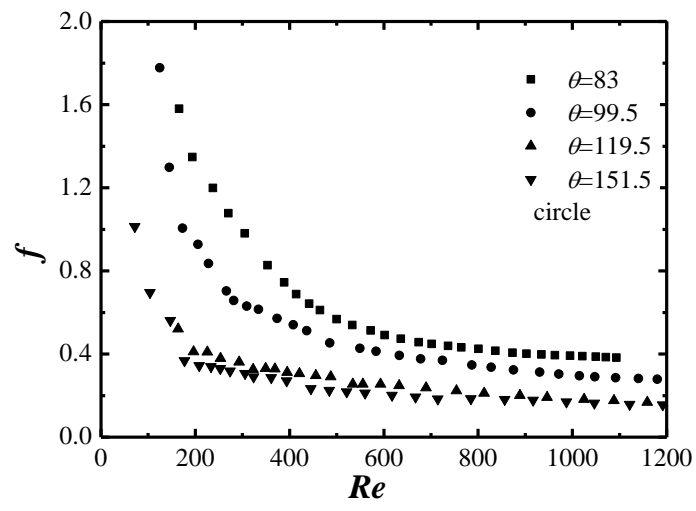

(c) circular micro pin fins

Fig. 8 The profiles of $f$ vs. Re in micro pin fins with different contact angles 
It can be seen from Fig. 8 that the values of $f$ are reduced with the increase of contact angle in all of the three micro pin fins, especially in the cases of low $R e$. This is related to the different microcosmic projections on surfaces with different contact angles, as shown in Fig.9.

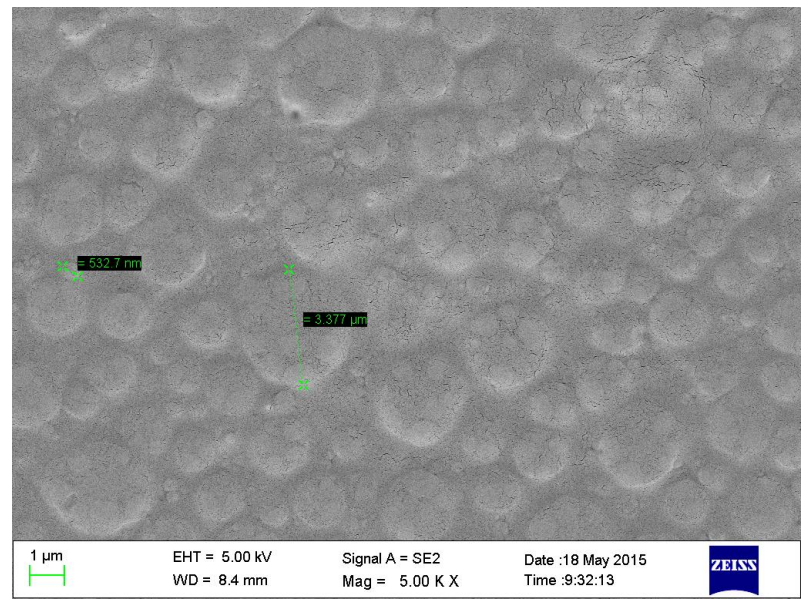

(a) $\theta=99.5^{\circ}, 5000 \mathrm{X}$

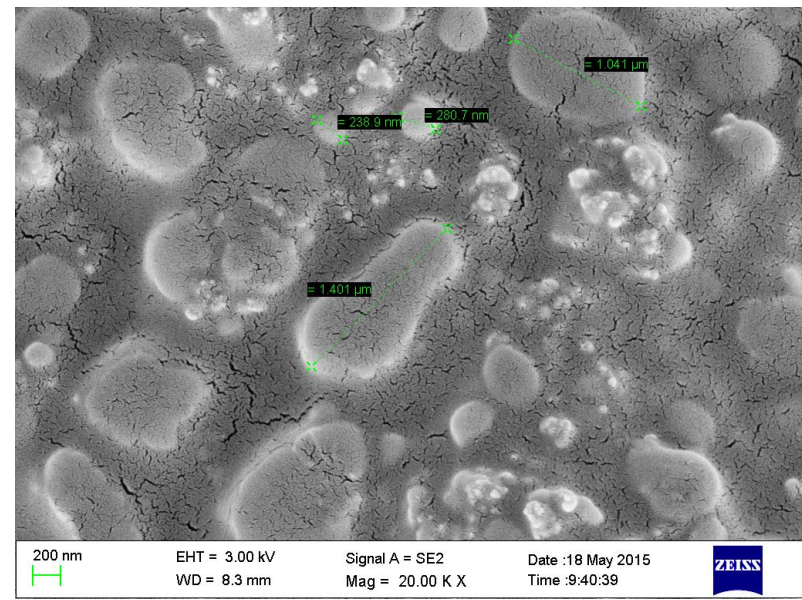

(b) $\theta=119.5^{\circ}, 20000 \mathrm{X}$

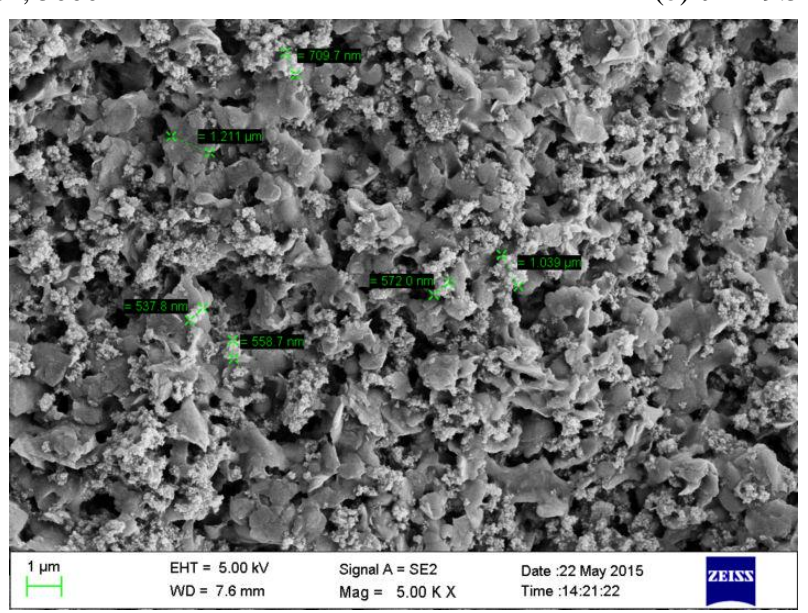

(c) $\theta=151.5^{\circ}, 5000 \mathrm{X}$

Fig.9 SEM photos of copper surfaces with $\theta=99.5^{\circ}, 119.5^{\circ}, 151.5^{\circ}$

The dimensions of nano projections and the spaces of the gaps are both reduced with the increase of the contact angle on the hydrophobic surfaces, so the total area of the solid-water interface is reduced while the working liquid flows through the hydrophobic micro pin fins. As a result, the frictional resistance in micro pin fins is reduced due to the slip flow on the air-water interface and the reduction of the velocity gradient in the boundary layer. However, this friction factor reduction is reduced with the increase of $R e$ attributed to the increase of the liquid pressure and the boundary layer separation, as shown in Fig.7. According to the hydrophobic model in Fig.6 (a), the differential pressure on the air-water interface can be calculated by the following equation:

$$
\Delta p=p_{\text {water }}-p_{\text {air }}=\frac{4 \sigma \cos (\pi-\phi)}{W}
$$

Keep increasing the differential pressure until the value of $\phi$ becomes as large as the contact angle $\theta$ in the micro pin fins, the Eq.(6) should be transformed into Eq. (7).

$$
\Delta p=p_{\text {water }}-p_{\text {air }}=\frac{4 \sigma \cos (\pi-\theta)}{W}
$$

If the differential pressure further increases, air will be extruded out of the gaps by the water, so the total area of 
the water-solid interface increases and the air-water interface area decreases. Consequently, the flow slip effect on hydrophobic walls in micro pin fins is weakened and the resistance reduction is reduced based on the present study.

In addition, the discrepancies of friction factors become small with the increase of $R e$ in elliptical, diamond and circular micro pins with different contact angles, but the discrepancies in circular micro pin fins are apparently larger than the those in elliptical micro pin fins at high $R e$, and the discrepancies in the diamond micro pin fins are in between. In authors' opinion, this phenomenon may be attributed to the boundary layer separation in the flow. For elliptical micro pin fins, the flow separation occurs lately compared with the other two types of micro pin fins due to the well streamlined shape, and the frictional resistance dominantly influences the value of the friction factor. Therefore, the flow resistance reduction due to the hydrophobic surfaces is more apparently impacted by the increase of the flow velocity in elliptical micro pin fins than that in the other micro pin fins. For the circular micro pin fins, the flow separates earlier and the areas of wake zones are larger than that in the elliptical and diamond micro pin fins at the same $R e$, so the effect of flow velocity on the resistance reduction in circular micro pin fins at high $R e$ is weakened and results in the phenomenon as shown in Fig.8.

Fig.10 illustrates the values of $d f_{-}$coe in elliptical, diamond and circular micro pin fins with different contact angles while $R e$ changes from $0-1200$.

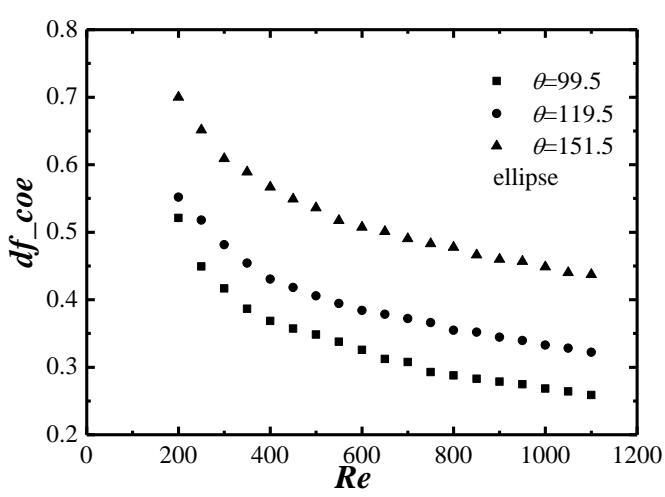

(a) elliptical micro pin fins

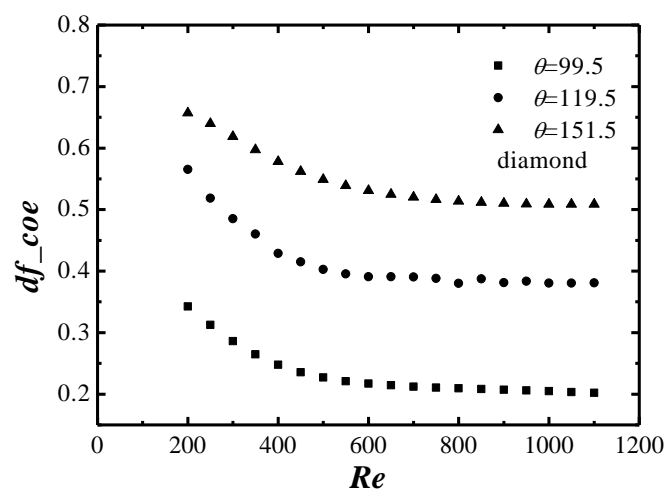

(b) diamond micro pin fins

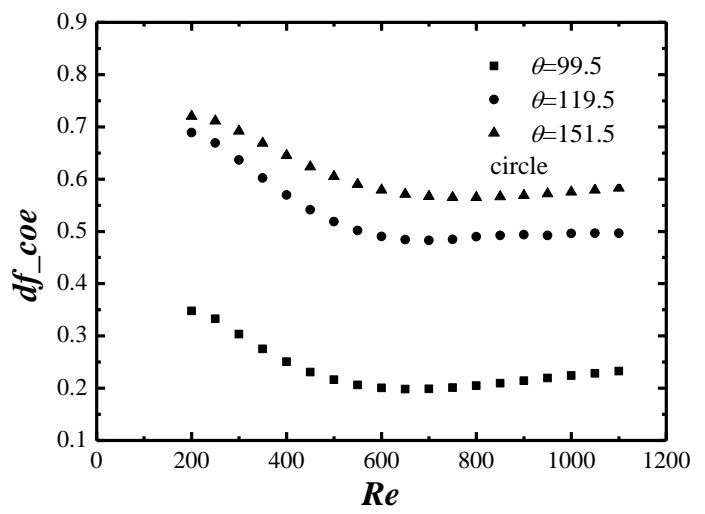

(c) circular micro pin fins

Fig. 10 Profiles of $d f_{-}$coe in micro pin fins with different contact angles with $R e$

It is known from Fig.10 that the values of $d f_{-}$coe become large with the increase of the contact angle in the three types of micro pin fins due to the air-water interfaces in the gaps among the nano projections on the hydrophobic surfaces. However, the discrepancies of $d f_{-}$coe between $\theta=119.5^{\circ}$ and $\theta=151.5^{\circ}$ in circular micro pin fins are much smaller than those in elliptical and diamond micro pin fins, as shown in Fig.10. Comparing the Fig.10(a) with Fig.7(a), Fig.10(b) with Fig.7(b) and Fig.10(c) with Fig.7(c), respectively, it can be known that 
$\theta=151.5^{\circ}$ is the optimal contact angle for the pump power and resistance reduction in elliptical and diamond micro pin fins, but the optimal contact angle in the circular micro pin fins is between $\theta=119.5^{\circ}$ and $\theta=151.5^{\circ}$ due to the simultaneous impact of contact angle increase and characteristic dimension change on the flow resistance in the circular micro pin fins.

Besides, it also can be seen from Fig.10 that the value of $d f_{-}$coe always decreases with $R e$ increasing from 0 to 1200 in elliptical micro pin fins with different contact angles, while the change rate of $d f_{-} c o e$ with Reis relatively small in diamond micro pin fins with $\theta=119.5^{\circ}$ and $\theta=151.5^{\circ}$ when $R e$ is larger than 400 . For circular micro pin fins with $\theta=119.5^{\circ}$ and $\theta=151.5^{\circ}$, the values of $d f_{-}$coe almost keep constants at $R e>400$. In authors' opinion, it may be attributed to the impact of hydrophobic surfaces on the flow transition from the laminar flow to the turbulent flow in wake zones of micro pin fins. The transition of the flow occurs earlier in wake zones of the diamond and the circular micro pin fins than that of the elliptical micro pin fins. The transitional flow in wake zones may result in high flow resistance in the circular micro pin fins compared with the elliptical and the diamond micro pin fins. However, this flow transition in circular micro pin fins is delayed by the increase of contact angle, and the pressure drag reduction is much larger than that of elliptical and diamond micro pin fins with streamlined shapes. Therefore, the reduction of frictional resistance due to the hydrophobic surfaces is reduced with the increase of $R e$, but the value of $d f_{-}$coe in circular micro pin fins does not decrease as apparent as that of the elliptical micro pin fins. The minimal values of $d f_{-}$coe are $50.81 \%$ and $58.68 \%$ in diamond and circular micro pin fins with contact angle of $\theta=151.5^{\circ}$, respectively.

\subsection{The influence of cross section shapes on the flow resistance reduction of the hydrophobic micro pin fins}

According to the discussion of Section 2.1, the flow resistance reduction in hydrophobic micro pin fins is related to the cross section shapes, thus in this part of work the pressure drop, the coefficients of pressure drop reductions, the friction factors and the coefficients of friction factor reductions will be analyzed in micro pin fins with different cross section shapes.

The pressure drops in hydrophobic micro pin fins with different cross section shapes are shown in Fig.11.

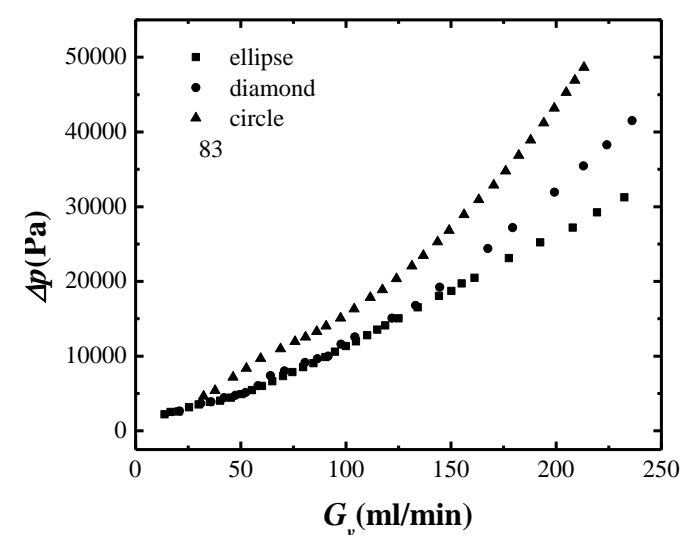

(a) $\theta=83^{\circ}$

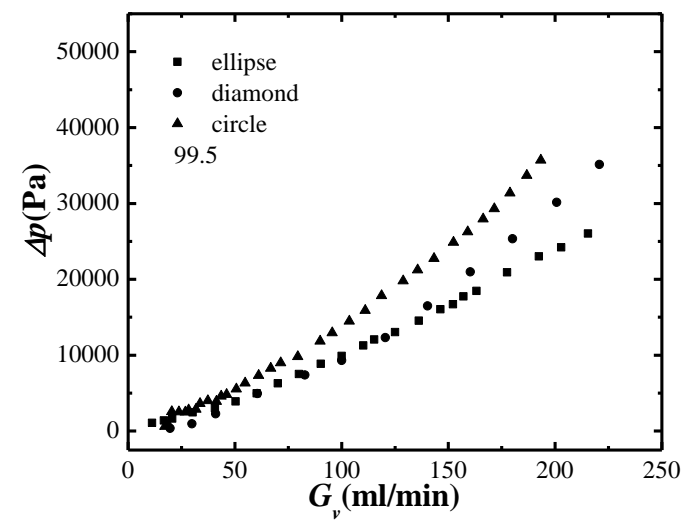

(b) $\theta=99.5^{\circ}$ 


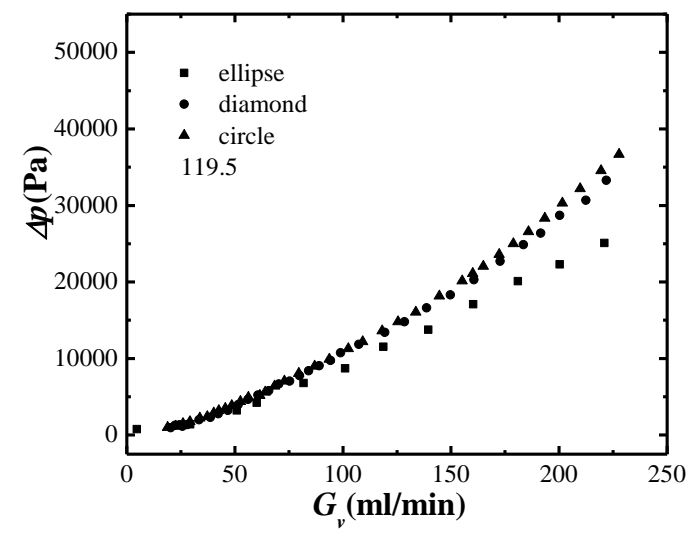

(c) $\theta=119.5^{\circ}$

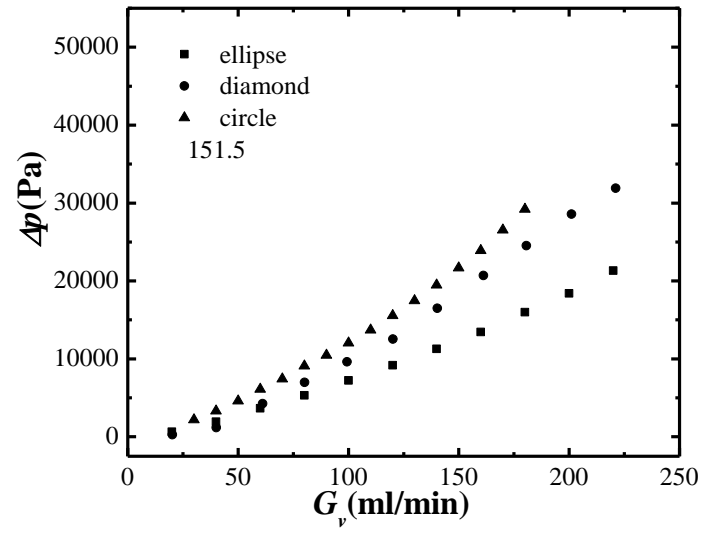

(d) $\theta=151.5^{\circ}$

Fig.11 The reductions of pressure drops in hydrophobic micro pin fins with different cross section shapes

It can be known that there is no apparent discrepancy of pressure drop among the micro pin fins with different cross section shapes and contact angles at low flow rate. However, the pressure drop becomes larger in diamond and circular micro pin fins than those in elliptical micro pin fins with the increase of the flow rate. This is attributed to the large pressure drag in the diamond and circular micro pin fins, especially in circular one with early flow separation. From the comparisons in Fig.11, it is found that the discrepancy of pressure drop between the diamond and the elliptical micro pin fins is smaller than that between the circular and the diamond micro pin fins with small contact angle, especially at low flow rate. However, the pressure drop in diamond micro pins becomes close to that in circular micro pin fins with the increase of the contact angle, which is attributed to the impact of the cross section shapes on the pressure drop reduction in hydrophobic micro pin fins. When the contact angle is small, the pressure drop reduction is not apparent due to the effect of hydrophobic surfaces, so the pressure drop in elliptical and diamond micro pin fins is close to each other because of the similar streamlined shapes, and both of them are lower than those in circular micro pin fins, especially at low flow rate. With the increase of the contact angle, the pressure drop reduction also increases, especially for the circular micro pin fins in which the pressure drag is more dominant in the total pressure drop. As discussed in Section 2.1, the pressure drag reduction due to the hydrophobic surface is not weakened as apparent as the frictional resistance reduction at high flow velocity, so the discrepancy of pressure drop between the circular and the diamond micro pin fins in Fig.11 (c)-(d) is apparently smaller than those in Fig.11(a)-(b).

In addition, it also can be seen from Fig.11 that the discrepancy of pressure drop between the diamond and the elliptical micro pin fins decreases at first and then increases, and so does the discrepancy between the circular and the diamond micro pin fins. This phenomenon is attributed to the change of the characteristic dimensions with the increase of the contact angle in the authors' opinion. As mentioned above, the characteristic dimension of micro pin fins becomes large with the increase of the contact angle, due to which the pressure drop reduction is weakened. It is also known from Fig.11 that the pressure drop is higher in circular micro pin fins than those in the other two types of test sections at the same contact angle, and the elliptical one is the smallest.

Fig.12 shows the values of $d p_{-} c o e$ in micro pin fins to analyze the impact of cross section shape on the pressure drop reduction with contact angles of $\theta=83^{\circ}, 99.5^{\circ}, 119.5^{\circ}$ and $151.5^{\circ}$, respectively. 


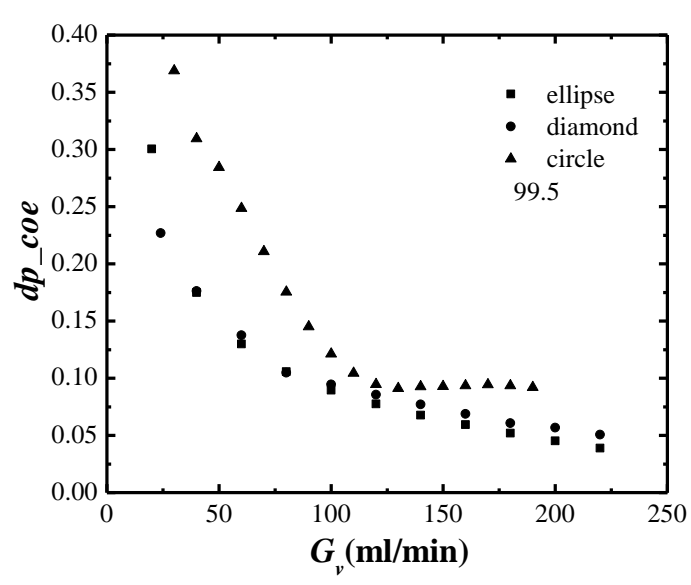

(a) $\theta=99.5^{\circ}$

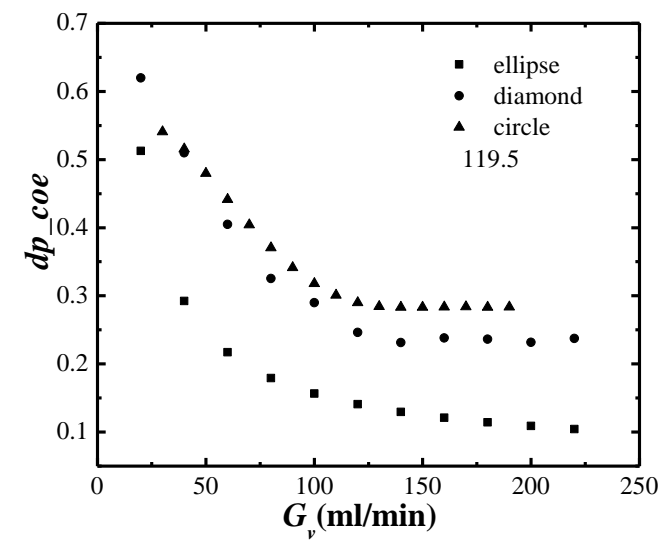

(b) $\theta=119.5^{\circ}$

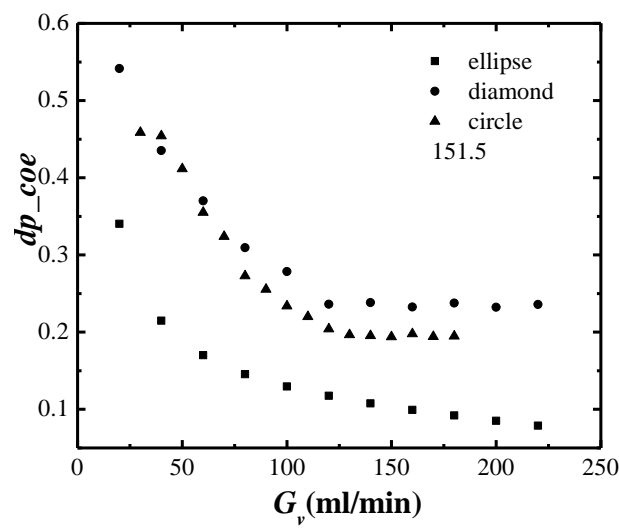

(c) $\theta=151.5^{\circ}$

Fig. 12 The relationships between the $d p \_c o e$ and the volume flow rate in micro pin fins with different cross section shapes

It can be known from the comparisons of Fig.12 that the discrepancies of $d p \_c o e$ between the diamond and the elliptical micro pin fins are small, especially at low flow rate. With the increase of the flow rate, the value of $d p \_c o e$ is reduced in diamond and in circular micro pin fins, while the value of former is slightly larger than that of the latter, as shown in Fig.12(a). When the contact angle is $99.5^{\circ}$, the value of $d p \_c o e$ is larger in circular micro pin fins than those in the diamond and the elliptical micro pin fins, which indicates that changing rate of pressure drop reduction in circular micro pin fins with contact angle increasing to $99.5^{\circ}$ is the larger than that of the diamond and the elliptical micro pin fins, although the pressure drop in circular micro pin fins is the largest at the same volume flow rate, as shown in Fig.12(a) and Fig.12(b). In authors' opinion, this is because that the impact of the hydrophobic surfaces is more apparent on the pressure drag reduction than that on the frictional resistance reduction in the flow across the micro pin fins. As mentioned above, the flow resistance in a channel is reduced by the hydrophobic surfaces because the water-solid interface area is decreased due to the nano scale projections on the hydrophobic surfaces, and the flow slip on the air-water interfaces in the gaps among the projections. As a result, the frictional resistance is reduced when the working fluid flows across the hydrophobic micro pin fins, correspondingly, the pressure drag is also reduced when the flow separation occurs. Furthermore, the flow separation is delayed due to the frictional resistance reduction with the increase of the contact angle, especially for the micro pin fin with early flow separation, which results in reduction of the wake zones area and the pressure drag. However, the frictional resistance is dominant in the total pressure drop instead of the pressure drag in elliptical and diamond micro pin fins with streamlined shapes, thus the impact of pressure drag reduction on the total pressure drop is weaker with the increase of the contact angle and the value of $d p \_c o e$ is smaller in elliptical and diamond micro pin fins than that in circular micro pin fins. 
When the contact angle increases from $99.5^{\circ}$ to $119.5^{\circ}$, the value of $d p \_c o e$ in circular micro pin fins is still the largest, as shown in Fig.12(b), while the discrepancies of $d p_{-}$coe between the diamond and the elliptical micro pin fins become larger compared with Fig.12(a), which is attributed to less streamlined shape of diamond micro pin fins than the elliptical ones. Therefore, the reduction of pressure drag results in more apparent total pressure drop reduction in diamond micro pin fins compared with the elliptical micro pin fins.

For the micro pin fins with contact angles of $151.5^{\circ}$, the value of $d p \_c o e$ is slightly smaller in circular micro pin fins than that in diamond micro pin fins but larger than that in elliptical ones as a results of the characteristic dimension change with the increase of the contact angle, as shown in Fig.12(c). Although the characteristic dimension becomes large with the increase of the contact angle, the change of characteristic dimension in circular micro pin fins not only increases the frictional resistance, but also results in an early flow separation, so the pressure drag in circular micro pin fins increases more quickly with the increase of flow rate compared with that in elliptical and diamond micro pin fins. Therefore, the value of $d p \_c o e$ with $\theta=151.5^{\circ}$ in circular micro pin fins is slightly lower than that in diamond micro pin fins under the comprehensive impacts of pressure drop reduction and the characteristic dimension change when the contact angle increasing from $83^{\circ}$ to $151.5^{\circ}$.

The comparisons of friction factors in micro pin fins with different cross section shapes at $\theta=83^{\circ}, 99.5^{\circ}, 119.5^{\circ}$ and $151.5^{\circ}$ are illustrated in Fig.13.

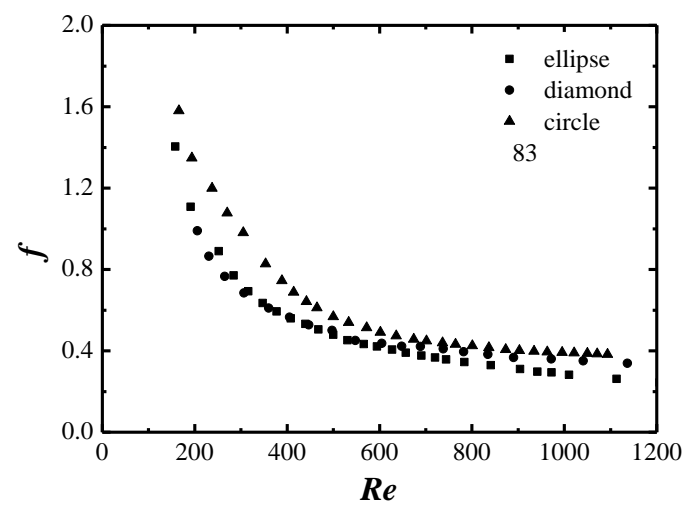

(a) $\theta=83^{\circ}$

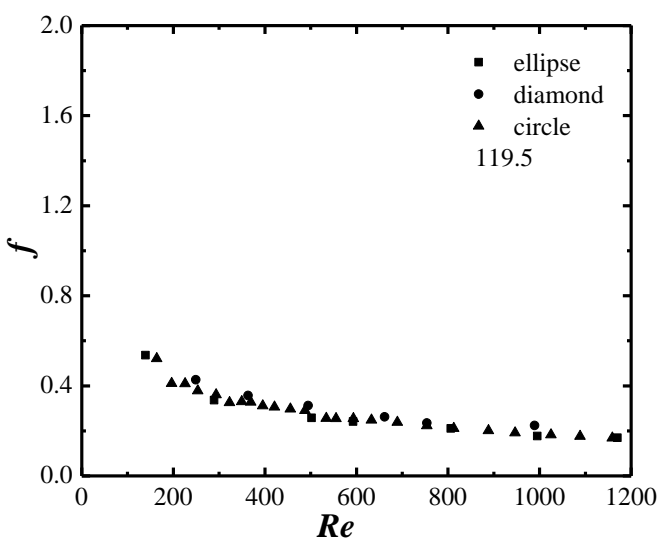

(c) $\theta=119.5^{\circ}$

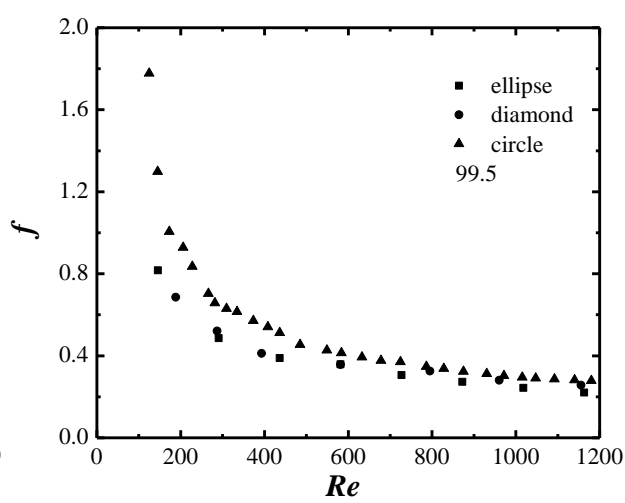

(b) $\theta=99.5^{\circ}$

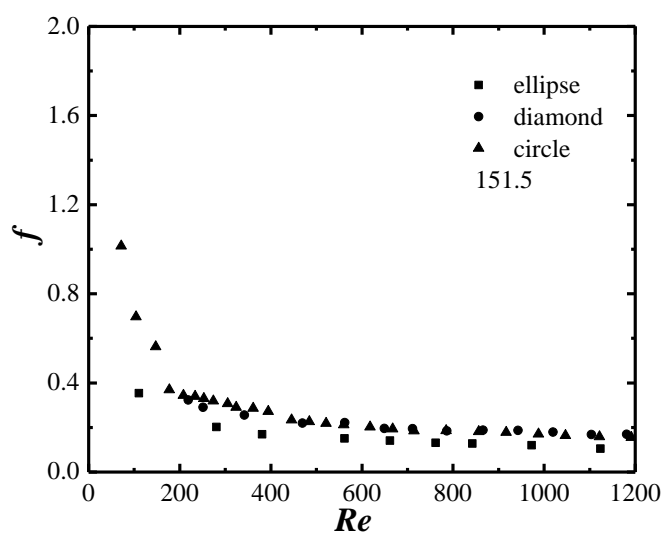

(d) $\theta=151.5^{\circ}$

Fig.13 The comparisons of friction factors in micro pin fins with different cross section shapes

It can be known from the comparisons that the friction factor is apparently higher in circular micro pin fins than that in the other two types of test sections, while the discrepancy of $f$ between the diamond and the elliptical micro pin fins is small at low $R e$, and then it becomes large with the increase of $R e$, as shown in Fig.13(a). In present research, the thickness of the boundary layer is comparable with the characteristic dimension of the pin fin in the flow across the micro pin fins, and the boundary layer shapes of the elliptical and diamond micro pin fins are 
similar, so the values of friction factors in these two micro pin fins are greatly close to each other. However, the flow separation of boundary layer occurs at lower $R e$ in the flow across the circular micro pin fins compared with the elliptical and the diamond test sections, as a result the impact of thick boundary layer on the friction factors of circular micro pin fins is weakened. Therefore, the value of $f$ is larger in circular micro pin fins than that in elliptical and diamond micro pin fins.

The values of $f$ are smaller in test sections with large contact angles than those in micro pin fins without hydrophobic surfaces, especially at high Re, as shown in Fig.13 (b), which is attributed to the impact of nano scale projections on the hydrophobic surfaces and influence of different cross section shapes on the resistance friction characteristics in micro pin fins. As discussed above, the existence of numerable nano projections on the hydrophobic surfaces not only result in the decrease of the solid-water interface area but also enhance the flow slip near the wall, thus the velocity gradients in boundary layer and the frictional resistance are reduced, and the flow separation is delayed and the pressure drag is reduced. When $R e$ and the pressure becomes large when the working liquid flows through the micro pin fins, the frictional resistance reduction is weakened, but the change of the pressure drag reduction is not as apparent as the frictional resistance. Therefore, the reduction of friction factors is more prominent in circular micro pin fins than that in elliptical and diamond micro pin fins, and the discrepancy of friction factor become small in the three micro pin fins with the increase of contact angle, as shown in Fig.13 (c)-(d).

In order to explore the effect of hydrophobic surfaces on the reduction of friction factors in micro pin fins with different cross section shapes, the comparisons of $d f_{-}$coe in elliptical, circular and diamond micro pin fins are illustrated in Fig. 14 when the contact angle of the flowing surface is $\theta=99.5^{\circ}, 119.5^{\circ}$ and $151.5^{\circ}$.

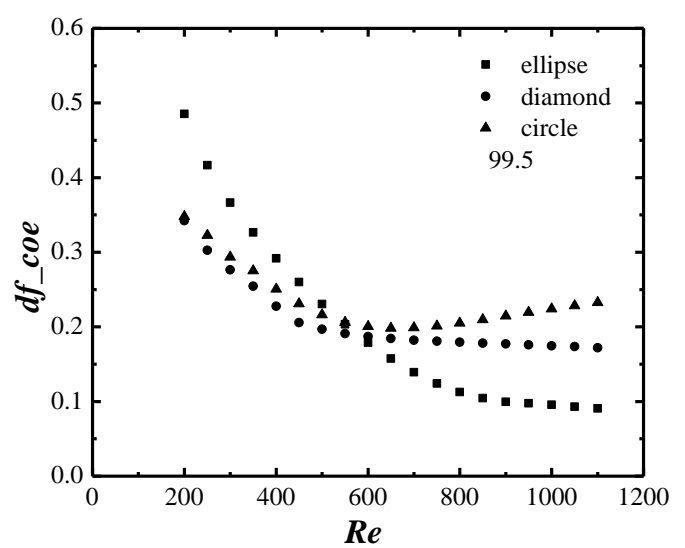

(a) $\theta=99.5^{\circ}$

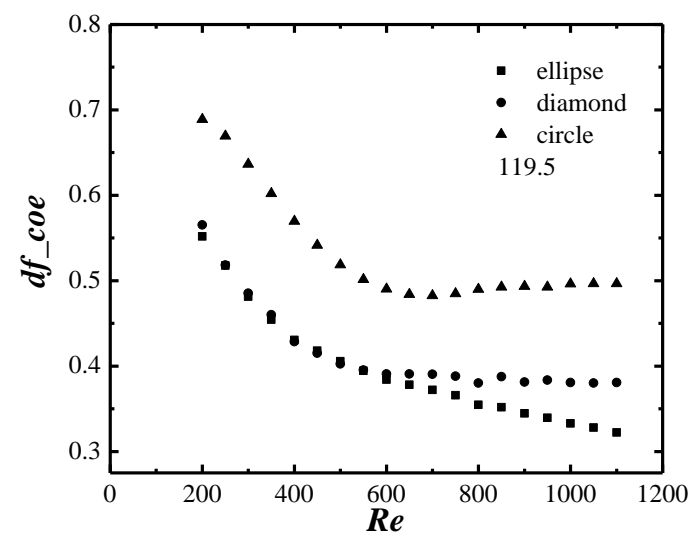

(b) $\theta=119.5^{\circ}$

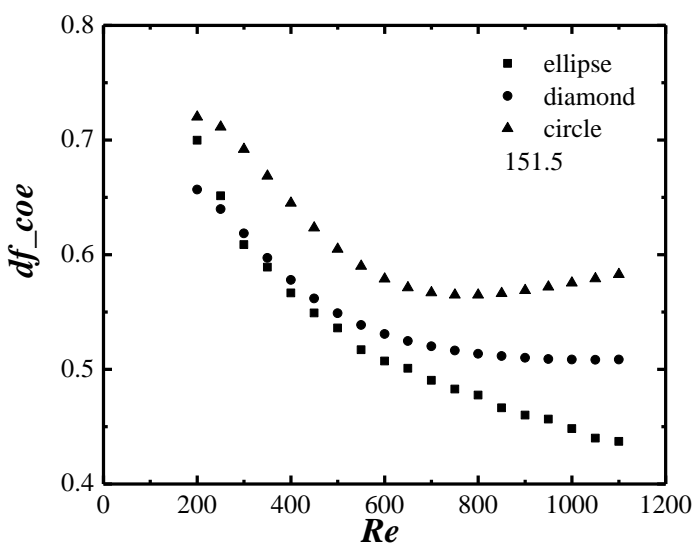

(c) $\theta=151.5^{\circ}$

Fig.14 Comparisons of $d f_{-}$coe with $R e$ in hydrophobic micro pin fins with different cross section shapes 
When the contact angle is $99.5^{\circ}$, the value of $d f_{-}$coe is higher in elliptical micro pin fins than that in diamond and the circular micro pin fins at low Re, as shown in Fig.14 (a), but it becomes the smallest among the three micro pin fins when $R e>600$, while the value of $d f_{-} c o e$ is larger in circular micro pin fins than that in diamond test sections. This phenomenon is attributed to the impact of hydrophobic surfaces on the boundary layer in the flow across the micro pin fins with different cross section shapes. As mentioned above, the flow resistance reduction in hydrophobic micro pin fins is weakened by the increase of the differential pressure and the flow velocity, however, the dimensions of the nano projections (the value of $W$ in Eq.(6)) are relatively large on the surfaces with small contact angle, hence the impact of the pressure on the frictional resistance reduction is more apparent in micro pin fins with small contact angle than that with large contact angle. In addition, the pressure is larger in circular micro pin fins than that in diamond and the elliptical micro pin fins; consequently, the value of $d f_{-} c o e$ is smaller in circular micro pin fins than that in elliptical micro pin fins at low Re. However, the pressure drag increases more quickly in circular micro pins than that in elliptical test sections with the increase of $R e$, which reduces the impact of pressure on the resistance reduction and delays the flow transition in the wake zones of the circular micro pin fins. Therefore, the total flow resistance is reduced apparently in circular micro pin fins at high $R e$, and the value of $d f_{-}$coe is larger in circular micro pin fins than those in elliptical micro pin fins, while the diamond is in between.

When the contact angle increases from $83^{\circ}$ to $119.5^{\circ}$ and $151.5^{\circ}$, the average distance among the nano projections on the surfaces becomes smaller than those in micro pin fins with $\theta=99.5^{\circ}$. As a result, the impact of pressure and velocity on the flow resistance reduction is weakened, and the rate of the frictional resistance to the total resistance is reduced. Especially for the circular micro pin fins, the flow resistances in wake zones are larger in it than those in elliptical and diamond micro pin fins; the increase of the contact angle may delay the boundary layer separation and the flow transition in wake zones. Therefore, the values of $d f_{-}$coe are larger in circular micro pin fins than those in elliptical and diamond micro pin fins with the contact angles of $119.5^{\circ}$ and $151.5^{\circ}$, as shown in Fig.14 (b)-(c). Likewise, the value of $d f_{-}$coe in diamond micro pin fins is very close to the that in elliptical micro pin fins at low $R e$, and then the former becomes larger than the latter with the increase of $R e$ attributed to the influence of flow separation on the flow resistance.

\section{Conclusions}

In present research, experimental investigations are carried out to measure the flow resistance characteristics in micro pin fins with different cross section shapes and different contact angles of $\theta=99.5^{\circ}, 119.5^{\circ}$ and $151.5^{\circ}$, respectively, and several conclusions are obtained as follows:

(1) The flow resistance in micro pin fins is significantly reduced by the hydrophobic surfaces, and the flow separation and transition in wake zones is delayed. The resistance reduction is more significant in the micro pin fins with early flow separation and high pressure drag in wake zones.

(2) When the contact angle increases from $83^{\circ}$ to $151.5^{\circ}$, the value of $d p \_c o e$ becomes large with the increase of the contact angle in elliptical and diamond micro pin fins; while for the circular micro pin fins, the value of $d p \_c o e$ becomes large with the contact angle increasing from $83^{\circ}$ to $119.5^{\circ}$, and then becomes small when contact angle increasing from $119.5^{\circ}$ to $151.5^{\circ}$.

(3) The value of $d p \_c o e$ always decreases with the increase of the flow rate in elliptical micro pin fins, but for diamond and circular micro pin fins it decreases at first and then becomes constant. The minimal value of $d p \_c o e$ is $22.92 \%$ in the diamond micro pin fins with corresponding contact angle of $\theta=151.5^{\circ}$ at high flow rate, but it is $28.35 \%$ in the circular micro pin fins when the contact angle is $\theta=119.5^{\circ}$.

(4) At the same $R e$, the value of $d f_{-}$coe becomes large with the increase of the contact angle in the micro pin fins with different cross section shapes. For a certain contact angle, the value of $d f_{-} c o e$ decreases with the increase of $R e$ in elliptical micro pin fins, but for diamond and the circular micro pin fins it decreases at first and then 
almost becomes constants. The minimal values of $d f_{-}$coe in diamond and circular micro pin fins are $50.81 \%$ and $58.68 \%$ with contact angle of $\theta=151.5^{\circ}$, respectively.

(5) At the same flow rate, the pressure drop in circular micro pin fins is the largest while the elliptical test section is the smallest. Increasing the contact angle to $151.5^{\circ}$, the value of $d p \_c o e$ in diamond micro pin fins becomes the largest while the elliptical test section is still the smallest.

(6) When the contact angle is $99.5^{\circ}$, the value of $d f_{-}$coe is larger in elliptical micro pin than that in circular and diamond micro pin fins at low $R e$. With the increase of the contact angle, the value of $d f_{-} c o e$ is apparently larger in circular micro pin fins than that in the other two test sections.

\section{Acknowledgement}

The authors acknowledge the support of National Science Foundation of China under Grant No.51306107, Science and Technology Development Planning Program No. 2014GGX104008.

\section{References}

[1] Ndao S., Peles Y., Jensen M.K., Effects of pin fin shape and configuration on the single-phase heat transfer characteristics of jet impingement on micro pin fins[J], International Journal of Heat and Mass Transfer, 70: 856-863, 2014.

[2] McGlen R.J., Jachuck R., Lin S., Integrated thermal management techniques for high power electronic devices[J], Applied Thermal Engineering, 25: 1143-1156, 2004.

[3] Kandlikar S.G., High flux heat removal with microchannels- a roadmap of challenges and opportunities[J], Heat Transfer Engineering, 2005, 25(8):5-14.

[4] Baudry J., Chariaix E., Tonck A., et al., Experimental evidence for a large slip effect at a nonwetting fluid-solid interface[J], Languir, 17(17): 5232-5236, 2001,

[5] Choi C.H., Johan K., Westin A., et al., Apparent slip flows in hydrophilic and hydrophobic microchannels[J], Physics of Fluids, 15(10): 2897-2902, 2003.

[6] Zhu Y.X., GRanick S., Limits of the hydrodynamic no-slip boundary condition[J], Physical Review Letter, 88(10): 106102-106105, 2002.

[7] Zhu X.Y., Zhu L., Chen, H.J., et al., Fabrication of multi-scale micro-lens arrays on hydrophobic surfaces using a drop-on-demand droplet generator[J], Optics \& laser Technology, 66: 156-165, 2015.

[8] Ou J., Blair P., Rothstein J.P., Laminar drag reduct-ion in microchannels using ultrahydrophobic surfaces[J], Physics of Fluids, 16 (12): 4635-4643, 2004.

[9] Ou J., Rothstein J.P., Direct velocity measurements of the flow past drag-reducing ultrahydrophobic surfa-ces[J], Physics of Fluids, 17: L1-L10, 2005.

[10] Tretheway D.C., Carl D. Meinhart, Apparent fluid slip at hydrophobic microchannel walls[J], Physics of Fluids, 14(3): L9-L12, 2002 .

[11] Hilpert M., Effects of dynamic contact angle on liquid infiltration into inclined capillary tubes: (Semi)-analytical solutions[J], Journal of Colloid and Interface Science, 337(1): 138-144, 2009.

[12] Mohammad N.N., Sekhavat S., Mofidi A., Drag reduction in a turbulent channel flow with hydro- phobic wall[J], Journal of Hydrodynamics, 24(3): 458-466, 2012.

[13] Yu D., Choi C., Kim M.H., Pressure drop and dynamic contact angle of triple-line motion in a hydrophobic microchannel[J], Experimental Thermal and Fluid Science, 39: 60-70, 2012. 
[14] Lyua S., Nguyenb D.C., Kima D., et al., Yoon Experimental drag reduction study of super-hydrophobic surface with dual-scale structures[J], Applied Surface Science, 286: 206-211, 2013.

[15] Wang B., Wang J.D., Dou Z.L., et al., Investigation of retention of gases in transverse hydrophobic microgrooved surfaces for drag reduction[J], Ocean Engineering, 79: 58-66, 2014.

[16] Li B.W., Yao Z.H., Hao P.F., Incompressible LBGK simulation of flow characteristics in a micro-channel with patterned superhydrophobic surfaces[J], Applied Mathematical Modelling, 39: 300-308, 2015.

[17] Mastrokalos M.E., Papadopoulos C.I., Kaiktsis L., Optimal stabilization of a flow past a partially hydrophobic circular cylinder[J], Computer \& Fluids, 107(1): 256-271, 2015.

[18] Cai Y.H., Hu J., Ma H.P., et al., Effects of hydrophilic/hydrophobic properties on the water behavior in the micro-channels of a proton exchange membrane fuel cell[J], Journal of Power Sources, 161(2): 843-848, 2006.

[19] Yin Y., Wu T.T., He P., et al., Numerical simulation of two-phase cross flow in microstructure of gas diffusion layer with variable contact angle[J], International Journal of Hydrogen Energy, 39(28): 15772-15785, 2014.

[20] Song M., Kim H.Y., Kim K., Effects of hydrophilic/hydrophobic properties of gas flow channels on liquid water transport in a serpentine polymer electrolyte membrane fuel cell[J], International Journal of Hydrogen Energy, 39: 19714-19721, 2014.

[21] Avcioglu G.S., Ficicilar B., Bayrakceken A., et al., High performance PEM fuel cell catalyst layers with hydrophobic channels[J], International Journal of Hydrogen Energy, In press, 2015.

[22] Sang Y.C., Ahmad B.A., Ala'a H.A., et al.,, Properties of super hydrophobic copper and stainless steel meshes: Applications in controllable water permeation and organic solvents/water separation[J], Applied Surface Science, 335(4): 107-114, 2015.

[23] Lenzinger M., Schweizer B., Two-phase flow equations with outflow boundary conditions in the hydrophobic hydrophilic case[J], Nolinear Analysis 73: 840-853, 2010.

[24] Choi Ch., Shin J.S., Yu D.I., et al., Flow boiling behaviors in hydrophilic and hydrophobic microchannels[J], Experimental Thermal and Fluid Science, 35: 816-824, 2011.

[25] Liu T.Y., Li P.L., Liu C.W.,et al., Boiling flow characteristics in microchannels with very hydrophobic surface to super-hydrophilic surface[J], International Journal of Heat and Mass Transfer, 54: 126-134, 2011.

[26] Cho S.C., Wang Y., Two-phase flow dynamics in a micro channel with heterogeneous surfaces[J], International Journal of Heat and Mass Transfer, 71: 349-360, 2014.

[27] Derby M.M., Chatterjee A., Peles Y., et al., Flow condensation heat transfer enhancement in a mini-channel with hydrophobic and hydrophilic patterns[J], International Journal of Heat and Mass Transfer, 68(1): 151-160, 2014.

[28] Moffat R.J., Describing the uncertainties in experimental results[J], Experimental Thermal and Fluid Science, 1: 3-17, 1988.

[29] Kosar A., Mishra C., Peles Y., Laminar flow across a bank of low aspect ratio micro pin fin[J], Journal of Fluids Engineering, 127:. 419-430, 2005. 